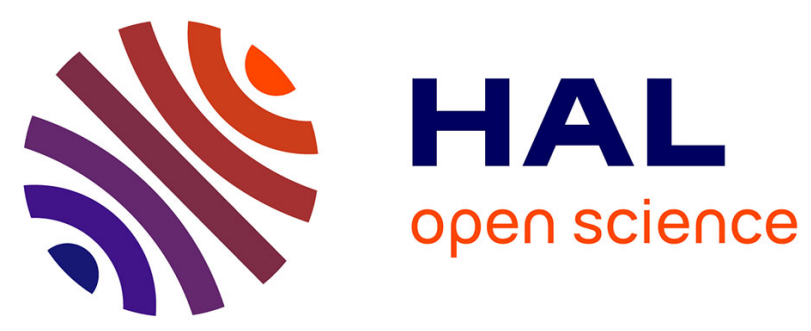

\title{
Natural element approximations involving bubbles for treating mechanical models in incompressible media
}

Julien Yvonnet, Pierre Villon, Francisco Chinesta

\section{To cite this version:}

Julien Yvonnet, Pierre Villon, Francisco Chinesta. Natural element approximations involving bubbles for treating mechanical models in incompressible media. International Journal for Numerical Methods in Engineering, 2006, 66 (7), pp.1125-1152. 10.1002/nme.1586 . hal-01007100

\section{HAL Id: hal-01007100 \\ https://hal.science/hal-01007100}

Submitted on 2 Apr 2017

HAL is a multi-disciplinary open access archive for the deposit and dissemination of scientific research documents, whether they are published or not. The documents may come from teaching and research institutions in France or abroad, or from public or private research centers.
L'archive ouverte pluridisciplinaire HAL, est destinée au dépôt et à la diffusion de documents scientifiques de niveau recherche, publiés ou non, émanant des établissements d'enseignement et de recherche français ou étrangers, des laboratoires publics ou privés.

\section{다)(1) $(5$}

Distributed under a Creative Commons Attribution - NonCommerciall 4.0 International 


\title{
Natural element approximations involving bubbles for treating mechanical models in incompressible media
}

\author{
J. Yvonnet ${ }^{1}$, P. Villon ${ }^{2}$ and F. Chinesta ${ }^{3 \S}$ \\ ${ }^{1}$ Université de Marne-la-Vallée, 5 Bd Descartes, 77454 Marne-la-Vallée Cedex, France \\ ${ }^{2}$ Université de Technlogies de Compiègne, BP 20529, 60205 Compiégne Cedex, France \\ ${ }^{3}$ École Nationale Supérieure d'Arts et Métiers, 151 boulevard de l'Hôpital, F-75013 Paris, France
}

In this paper, a new approach is proposed to address issues associated with incompressibility in the context of the meshfree natural element method (NEM). The NEM possesses attractive features such as interpolant shape functions or auto-adaptive domain of influence, which alleviates some of the most common difficulties in meshless methods. Nevertheless, the shape functions can only reproduce linear polynomials, and in contrast to moving least squares methods, it is not easy to define interpolations with arbitrary approximation consistency. In order to treat mechanical models involving incompressible media in the framework of mixed formulations, the associated functional approximations must satisfy the well-known inf-sup, or LBB condition. In the proposed approach, additional degrees of freedom are associated with some topological entities of the underlying Delaunay tessellation, i.e. edges, triangles and tetrahedrons. The associated shape functions are computed from the product of the NEM shape functions related to the original nodes. Different combinations can be used to construct new families of NEM approximations. As these new approximations functions are not related to any node, as they vanish at the nodes, from now on we refer these shape functions as bubbles. The shape functions can be corrected enforcing different reproducing conditions, when they are used as weights in the moving least square (MLS) framework. In this manner, the effects of the obtained higher approximation consistency can be evaluated. In this work, we restrict our attention to the $2 \mathrm{D}$ case, and the following constructions will be considered: (a) bubble functions associated with the Delaunay triangles, called b1-NEM and (b) bubble functions associated with the Delaunay edges, called $b 2-N E M$. We prove that all these approximation schemes allow direct enforcement of essential boundary conditions. The bubble-NEM schemes are then used to approximate the displacements in the linear elasticity mixed formulation, the pressure being approximated by the standard NEM. The numerical LBB test is passed for all the bubble-NEM approximations, and pressure oscillations are removed in the incompressible limit. 
KEY WORDS: meshless methods; natural element method; bubble functions; mixed formulations; incompressible media; LBB condition

\section{INTRODUCTION}

It is well known that the solution of mechanical problems involving incompressible media using the standard displacement-based finite element technique may yield solutions, which are grossly in error [1]. The difficulty is that the computed displacement field needs to satisfy the constraint of very small volumetric strains (which become zero as the condition of total incompressibility is approached) while the pressure is of the order of the boundary tractions. The displacement approximation space is not rich enough to accommodate this constraint without a drastic reduction in the rate of convergence, also known as locking [1].

For the analysis of such problems, one solution is to use a mixed formulation in which different approximation spaces are used for the displacement and pressure fields interpolation. Although numerous mixed formulations may be developed, only those that are stable are useful in practice [2,3].The solvability, stability and optimality of mixed formulations are related to a compatibility condition, the so-called LBB (or inf-sup) condition [4]. The analytical proof whether the inf-sup condition is satisfied for a specific formulation is, however, difficult, and this has spurred the use of a numerical inf-sup test [4-6].

Meshless methods have been developed over the past decade to circumvent some difficulties associated with the finite element method. One of the advantages of meshfree methods over mesh-based techniques is that they can often handle geometric changes like free surfaces or large deformation better. Different methods can be found, such as the diffuse element method (DEM) [7], the element-free Galerkin method (EFG) [8], the reproducing kernel particle method [9], SPH and radial basis functions methods [10,11], or the natural element method (NEM) [12]. Accounting incompressibility in meshless methods is still an open topic. Until recently, it was stated that meshfree methods are immune to locking $[8,13]$. Furthermore, the EFG has been actually proposed for treating isochoric elastoplasticity by considering the shape functions support large enough [14]. In the context of the RKPM, a similar claim was made in the context of large deformation of nearly incompressible hyperelastic [15] and elastoplastic materials [16]. Recently, it has been reported that meshfree methods are in fact not locking-free in the incompressibility limit [17]. In a recent paper [18] this issue is clarified determining the influence of the EFG shape functions support on the locking behaviour. The main conclusion was that by increasing the shape functions support the looking can be attenuated, but never suppressed. Several attempts have been proposed to avoid locking in the context of meshfree methods. Huerta et al. [19] developed a so-called pseudo-divergence-free approximation, consisting in using approximation functions that verify approximately the divergence-free constraint for a given discretization in a diffuse sense. Dolbow and Belytschko [17] have proposed a mixed displacement/pressure formulation and selective reduced integration to alleviate locking. Chen et al. note that the use of large support size is computationally expensive and, moreover, cannot remove pressure oscillations [20]. They proposed a pressure projection combined with a reduced integration to remove pressure oscillations in nearly incompressible elasticity problems. The NEM is a novel meshfree method. Its attractive features are: (a) interpolant character of 
the shape functions; (b) strict linearity of the shape functions over the boundaries, ${ }^{\text {Il }}$ and (c) the support of the shape functions is based on the Delaunay spheres of the surrounding nodes, and automatically adapts to the local nodal density. Properties (a) and (b) allow direct enforcement of the essential boundary conditions and guarantees conforming approximations in presence of interfaces [23]. Property (c) allows simple refinement strategies [24]. However, unlike the moving least square (MLS) techniques, it is not possible to directly enrich the basis in order to improve the reproducing conditions (approximation consistency). Sukumar [25] was the first to propose a mixed NEM interpolation using constant piecewise shape functions for the pressure approximation, and the standard NEM for the approximation of the displacements. González et al. [26] proposed an enrichment of the NEM in the context of the partition of unity paradigm [27] to construct richer approximations, in order to verify the inf-sup condition. Chen et al. [28] proposed to use a stabilized nodal integration to avoid locking in near-incompressible elastostatics. In this paper we propose a new approach in the context of the NEM allowing to define stable mixed formulations for treating mechanical models involving incompressible media. In the proposed technique, additional degrees of freedom associated with some topological entities of the underlying Delaunay tessellation, i.e. edges, triangles and tetrahedrons are introduced. The associated bubble shape functions are computed from the product of the NEM shape functions related to the generating nodes of the entity. Moreover, we have also tested the use of the shape functions as weights in a MLS framework in order to enforce a certain degree of consistency of the functional approximation (e.g. quadratic consistency). The effect of the higher consistency will then be evaluated. The organization of this paper is as follows. In Section 2 we review the interpolation scheme used in the displacement-based NEM. In Section 3 we introduce the bubble-NEM approximation, focusing on two particular 2D cases using bubble functions associated with (a) the Delaunay triangles, called b1-NEM and (b) with the edges of the Delaunay triangles, called $b 2-N E M$. It is proved that essential boundary conditions can be enforced directly in both cases. In Section 4 we introduce a displacement/pressure mixed formulation for the analysis of problems involving linear elasticity in the context of the b-NEM. In Section 5 we discuss the inf-sup condition, and analyse the stability of the different displacement/pressure discretization schemes previously introduced in the incompressible limit.

\section{REVIEW OF THE NATURAL ELEMENT METHOD}

\subsection{Natural neighbour interpolation}

We briefly touch upon the foundation of Sibson's natural neighbour co-ordinates (shape functions) that are used in the NEM. For a more in-depth discussion on the Sibson interpolant and its application for solving second-order partial differential equations, the interested reader can refer to References [12,29]. The NEM interpolant is constructed on the basis of the Voronoi diagram (see Figure 1). The Delaunay tessellation is the topological dual of the Voronoi diagram.

\footnotetext{
${ }^{\mathbb{I}}$ This property is restricted to convex boundaries [12]. However, some techniques have been provided to extend it to non-convex boundaries [21,22].
} 


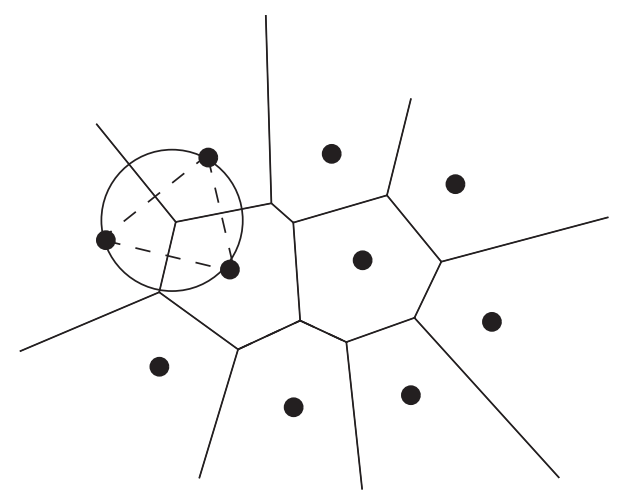

Figure 1. Voronoi diagram, Delaunay triangle and Delaunay circle.
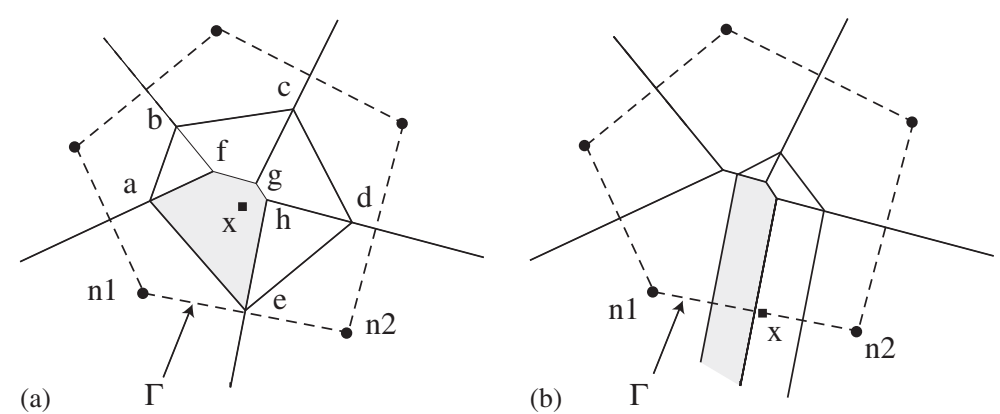

Figure 2. Construction of the Sibson shape functions.

Consider a set of nodes $S=\left\{n_{1}, n_{2}, \ldots, n_{N}\right\}$ in $\mathfrak{R}^{\mathrm{dim}}$. The Voronoi diagram is the subdivision of $\Re^{\text {dim }}$ into regions $T_{i}$ (Voronoi cells) defined by

$$
T_{i}=\left\{\mathbf{x} \in \Re^{\operatorname{dim}}: d\left(\mathbf{x}, \mathbf{x}_{i}\right)<d\left(\mathbf{x}, \mathbf{x}_{j}\right) \forall j \neq i\right\} \quad \forall i
$$

The Sibson co-ordinates of $\mathbf{x}$ with respect to a natural neighbour $n_{i}$ (see Figure 2) is defined as the ratio of the overlap area (volume in 3D) of their Voronoi cells to the total area (volume in $3 \mathrm{D}$ ) of the Voronoi cell related to point $\mathbf{x}$. If we consider the $2 \mathrm{D}$ example depicted in Figure 2(a), we have

$$
\phi_{1}(\mathbf{x})=\frac{\operatorname{Area}(a f g h e)}{\operatorname{Area}(a b c d e)}
$$

If point $\mathbf{x}$ coincides with the node $n_{i}$, i.e. $\left(\mathbf{x}=\mathbf{x}_{i}\right), \phi_{i}\left(\mathbf{x}_{i}\right)=1$, and all other shape functions are zero, i.e. $\phi_{j}\left(\mathbf{x}_{i}\right)=\delta_{i j}\left(\delta_{i j}\right.$ being the Kronecker delta), then the properties of positivity, interpolation, and partition of unity are then verified [12]:

$$
0 \leqslant \phi_{i}(\mathbf{x}) \leqslant 1
$$




$$
\begin{aligned}
\phi_{i}\left(\mathbf{x}_{j}\right) & =\delta_{i j} \\
\sum_{i=1}^{n} \phi_{i}(\mathbf{x}) & =1
\end{aligned}
$$

The natural neighbour shape functions also satisfy the local co-ordinate property [30], namely,

$$
\mathbf{x}=\sum_{i=1}^{n} \phi_{i}(\mathbf{x}) \mathbf{x}_{i}
$$

which combined with Equation (3), implies that the natural neighbour interpolant spans the space of linear polynomials (linear completeness).

Sibson natural neighbour shape functions are $C^{1}$ at any point except at the nodes, where they are only $C^{0}$. The $C^{1}$ continuity everywhere can be obtained by using special classes of natural neighbour shape functions [31].

The support (domain of influence) of a shape function $\phi_{i}$ is the union of the Delaunay spheres (circumscribing the Delaunay tetrahedrons) containing the node $n_{i}$. This support is thus not radial and automatically adapts to the relative position of $n_{i}$ and its neighbours, whether it is the density or the regularity of the nodal distribution.

Another important property of this interpolant is its strict linearity over the boundary of convex domains. The proof can be found in Reference [12]. An illustration is depicted in Figure 2(b): as the areas associated to points on the boundary become infinite, the contribution of internal points vanish in the limit when the point approaches the convex boundary, and the shape functions associated with nodes $n_{1}$ and $n_{2}$ become linear on the segment $\left(n_{1}-n_{2}\right)$. This is not true in the case of non-convex boundaries, and an appropriate treatment must be introduced to preserve this property in non-convex domains [21,22]. Essential boundary conditions can thus be enforced directly, as in the finite element method. This property also guarantees strict continuity of the approximation across material interfaces [23], which is an issue in most meshfree methods.

Consider an interpolation scheme for a vector-valued function $\mathbf{u}(\mathbf{x}): \Omega \subset \mathfrak{R}^{2} \rightarrow \Re$, in the form

$$
\mathbf{u}^{h}(\mathbf{x})=\sum_{i=1}^{n} \phi_{i}(\mathbf{x}) \mathbf{u}_{i}
$$

where $\mathbf{u}_{i}$ are the nodal values of the field at the $n$ natural neighbour nodes, and $\phi_{i}(\mathbf{x})$ are the shape functions associated with each neighbour node. It is noted that Equation (5) defines a local interpolation scheme. Thus, the trial and test functions used in the discretization of the variational formulation describing the problems treated in this paper take the form of Equation (5).

One of the drawbacks of the NEM is that natural neighbour shape functions can only reproduce at best linear fields, which induces difficulties to construct mixed formulations, where the different fields must be approximated in different approximation spaces in order to avoid numerical locking (LBB condition [4]). In the next section, a new approach is proposed to enrich the NEM approximation. 


\section{HIERARCHICAL BUBBLE FUNCTIONS IN THE NATURAL ELEMENT METHOD}

Consider an open bounded domain $\Omega \in \mathfrak{R}^{\mathrm{dim}}$ with boundary $\Gamma$, dim being the space dimension. Assume that $\Omega$ is discretized by a set of nodes $S$. Let $D(S)$ the simplicial complex associated with the Delaunay tesselation of $S$. A simplicial complex $K$ in $\Re^{\text {dim }}$ is a collection of simplices (hypertetrahedra) in $\Re^{\mathrm{dim}}$ such that

(i) every face of a simplex $K$ is in $K$; and

(ii) the intersection of any two simplices of $K$ is a face of each of them [32].

If we denote $F_{k}$ the set of $k$-simplices $(0 \leqslant k \leqslant 3)$, in $R^{3}$ the Delaunay tessellation $D(S)$ will be defined as the simplicial complex defined by the tetrahedra in $F_{3}$, the triangles in $F_{2}$, the edges in $F_{1}$, and the vertices in $F_{0}$. We denote these collections $T(S), F(S), E(S)$ and $V(S)$, respectively.

In order to construct richer approximations, new shape functions can be associated with the different $k$-simplices. The case $1<k<3$ is related to the concept of hierarchical methods [33]. The concept of hierarchical bubble shape functions is a very simple way to construct richer approximations. The extension to meshfree methods is not an easy matter in general, in the absence of topology related to some elements. In the natural element, the underlying Delaunay triangulation allows the use of such approach.

The key idea is to associate new shape functions to the $k$-simplices of the Delaunay tessellation, i.e. tetrahedra $T^{\prime} \in T(S)$, triangular facets $F^{\prime} \in F(S)$ and edges connecting two nodes in the Delaunay triangulation $E^{\prime} \in E(S)$.

\section{1. b-NEM approximation}

A $k$-simplex (K-S) (vertex, edge, triangular facet or tetrahedron) is generated by $K=k+1$ vertices $\left(k=0,1,2\right.$ and 3 , respectively). The bubble shape function of an entity $\chi_{j}$ generated by $K$ vertices is computed as

$$
\phi_{j}^{*}(\mathbf{x})=\prod_{p=1}^{K} \phi_{p}(\mathbf{x})
$$

where $\phi_{p}(\mathbf{x})$ is the NEM shape function (Equation (2)) associated with node $n_{p}$ computed at point $\mathbf{x}$.

The support (domain of influence) of a K-S generated by $K$ vertices (nodes) in $S$ is the union of the Delaunay spheres containing the $K$ nodes. It results, in 2D:

(i) if $\chi_{j}$ is a Delaunay triangle $(\chi \in F(S))(k=2)$, the support of $\chi_{j}$ is composed with one circle containing the 3 generating nodes of the triangle (see Figure 3(a)); and

(ii) if $\chi_{j}$ is an edge of a Delaunay triangle $(\chi \in E(S))(k=1)$, the support of $\chi_{j}$ is composed with the union of two circles (if $\chi_{j} \notin \Gamma$ ), or one circle if $\chi_{j} \in \Gamma$ (see Figure 3(b)), containing the 2 generating nodes of $\chi_{j}$.

We now consider the following approximation scheme:

$$
\mathbf{u}^{h}(\mathbf{x})=\sum_{i=1}^{n} \phi_{i}(\mathbf{x}) \mathbf{u}_{i}+\sum_{j=1}^{m} \phi_{j}^{*}(\mathbf{x}) \gamma_{j}
$$




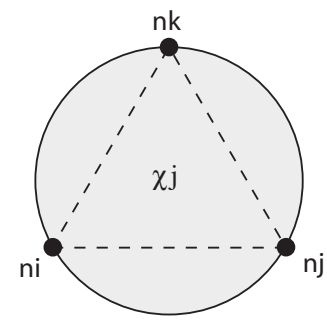

(a)

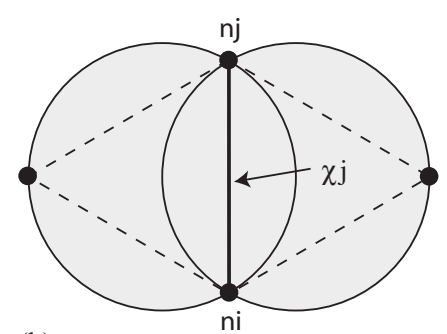

(b)

Figure 3. Supports of the bubble shape functions associated with the Delaunay $k$-simplex: (a) support of a Delaunay triangle $\chi_{j} n_{i}-n_{j}-n_{k}$; and (b) support of a Delaunay edge $\chi_{j} n_{i}-n_{j}$.

where $n$ is the number of natural neighbours of point $\mathbf{x}, \phi_{i}(\mathbf{x})$ is the NEM shape function related to node $n_{i} \in S$ computed at point $\mathbf{x}, \phi_{j}^{*}(\mathbf{x})$ is the bubble shape function defined in Equation (6) associated with the $m$ influent $\mathrm{K}-\mathrm{S}$, and $\gamma_{j}$ is an additional degree of freedom (d.o.f.).

Remarks

(i) Different combinations can be chosen for enriching the approximation, i.e. using only bubble functions associated with the edges, with the Delaunay triangles, or both. In three dimensions, the number of possibilities is higher, as three topological entities can be considered, i.e. edges, triangular facets and tetrahedrons. In this paper, we only focus on the $2 \mathrm{D}$ case.

(ii) The evaluation of the bubble shape functions associated with the K-S is not costly as it only requires the product of available NEM shape functions computed at point $\mathbf{x}$.

(iii) The enrichment with one bubble function associated with each Delaunay triangle is similar from the construction point of view to the MINI element [34], when one uses the NEM shape functions instead of FEM shape functions.

(iv) Despite that the approximation scheme defined in Equation (7) is richer than standard NEM approximation and can be used in mixed formulation, it does not satisfy any reproducing property other than the linear consistency. A correction of these shape functions in a MLS framework is proposed in the next section.

In this paper, two approximation schemes are investigated and compared: (a) one using bubble functions associated with the Delaunay triangles (that we call b1-NEM); and (b) one using bubble functions associated with the Delaunay edges (called b2-NEM).

\section{2. $b$-NEM with reproducing properties}

In this section we proceed to correct the shape functions previously constructed defining the approximation scheme (7) within a standard MLS framework, in order to evaluate the benefits provided by the higher approximation consistency. We briefly summarize the MLS procedure $[7,8]$. Let $w_{i}(\mathbf{x})$ some weight function either associated with a standard or a bubble-NEM 
shape function, computed at point $\mathbf{x}$. Let the following approximation scheme:

$$
\mathbf{u}^{h}(\mathbf{x})=\mathbf{p}^{\mathrm{T}}(\mathbf{x}) \mathbf{a}(\mathbf{x})
$$

with $\mathbf{p}^{\mathrm{T}}(\mathbf{x})$ a polynomial basis, i.e. $\mathbf{p}^{\mathrm{T}}(\mathbf{x})=[1, x, y, x y]$ and $\mathbf{p}^{\mathrm{T}}(\mathbf{x})=\left[1, x, y, x y, x^{2}, y^{2}\right]$ for a bilinear and quadratic basis, respectively, in $2 \mathrm{D}$, and $\mathbf{a}(\mathbf{x})$ a vector of unknown coefficients. In order to determine $\mathbf{a}(\mathbf{x})$, the functional $J$ defined by Equation (9) has to be minimized with respect to $\mathbf{a}(\mathbf{x})$ :

$$
J=\frac{1}{2} \sum_{i=1}^{n} w_{i}(\mathbf{x})\left[\mathbf{p}^{\mathrm{T}}\left(\mathbf{x}_{i}\right) \mathbf{a}(\mathbf{x})-u_{i}\right]^{2}
$$

where $u_{i}$ are the nodal unknown associated with neighbours of point $\mathbf{x}$. The minimization of $J$ with respect to the unknown coefficient $a_{j}(\mathbf{x})$ leads to

$$
\frac{\partial J}{\partial a_{j}(\mathbf{x})}=\sum_{k=1}^{n} a_{k}\left[\sum_{i=1}^{n} w_{i}(\mathbf{x}) p_{j}\left(\mathbf{x}_{i}\right) p_{k}\left(\mathbf{x}_{i}\right)\right]-\sum_{i=1}^{n} w_{i}(\mathbf{x}) p_{j}\left(\mathbf{x}_{i}\right) u_{i}=0
$$

which leads to the linear system

$$
\mathbf{A}(\mathbf{x}) \mathbf{a}(\mathbf{x})=\mathbf{B}(\mathbf{x}) \mathbf{u}
$$

where the matrix $\mathbf{A}(\mathbf{x})$ and $\mathbf{B}(\mathbf{x})$ are defined by

$$
\begin{aligned}
A_{j k}(\mathbf{x}) & =\sum_{i=1}^{n} w_{i}(\mathbf{x}) p_{j}\left(\mathbf{x}_{i}\right) p_{k}\left(\mathbf{x}_{i}\right) \\
B_{i j}(\mathbf{x}) & =w_{i}(\mathbf{x}) p_{j}\left(\mathbf{x}_{i}\right)
\end{aligned}
$$

Substituting $\mathbf{a}(\mathbf{x})$ in Equation (8), it results

$$
u^{h}(\mathbf{x})=\mathbf{p}^{\mathrm{T}}(\mathbf{x}) \mathbf{A}^{-1}(\mathbf{x}) \mathbf{B}(\mathbf{x}) \mathbf{u}
$$

By identification, the new shape functions are given by

$$
\psi^{\mathrm{T}}(\mathbf{x})=\mathbf{p}^{\mathrm{T}}(\mathbf{x}) \mathbf{A}^{-1}(\mathbf{x}) \mathbf{B}(\mathbf{x})
$$

The reproducing b-NEM shape functions are computed by setting $w_{i}(\mathbf{x})=\left\{\phi_{i}(\mathbf{x}) ; \phi_{j}^{*}(\mathbf{x})\right\}$, $\phi_{i}(\mathbf{x})$ and $\phi_{j}^{*}(\mathbf{x})$ being the shape functions defined in (2) and (6).

In the following, $\psi(\mathbf{x})$ is a vector containing the shape functions associated with influent nodes or K-S at point $\mathbf{x}$. The properties of the resulting shape functions are discussed in Section 3.3.

The shape functions derivatives are obtained from

$$
\frac{\partial \boldsymbol{\psi}(\mathbf{x})}{\partial \mathbf{x}}=\frac{\partial \mathbf{p}^{\mathrm{T}}(\mathbf{x})}{\partial \mathbf{x}} \mathbf{A}^{-1}(\mathbf{x}) \mathbf{B}(\mathbf{x})+\mathbf{p}^{\mathrm{T}}(\mathbf{x}) \mathbf{A}^{-1}(\mathbf{x}) \frac{\partial \mathbf{B}(\mathbf{x})}{\partial \mathbf{x}}-\mathbf{p}^{\mathrm{T}}(\mathbf{x}) \mathbf{A}^{-1}(\mathbf{x}) \frac{\partial \mathbf{A}(\mathbf{x})}{\partial \mathbf{x}} \mathbf{A}^{-1} \mathbf{B}(\mathbf{x})
$$


where

$$
\begin{aligned}
& \frac{\partial A_{j k}(\mathbf{x})}{\partial \mathbf{x}}=\sum_{i=1}^{n} \frac{\partial w_{i}(\mathbf{x})}{\partial \mathbf{x}} p_{j}\left(\mathbf{x}_{i}\right) p_{k}\left(\mathbf{x}_{i}\right) \\
& \frac{\partial B_{i j}(\mathbf{x})}{\partial \mathbf{x}}=\frac{\partial w_{i}(\mathbf{x})}{\partial \mathbf{x}} p_{j}\left(\mathbf{x}_{i}\right)
\end{aligned}
$$

It should be noted that evaluation of (17) and (18) implies the computation of the derivatives of the NEM shape functions. A closed form for the Sibson shape functions derivatives have been provided by Piper [35]. Sibson shape function (related to node $i$ and evaluated at point $\mathbf{x}$ ) can be written as

$$
\phi_{i}(\mathbf{x})=\frac{\alpha_{i}(\mathbf{x})}{\sum_{j=1}^{n} \alpha_{j}(\mathbf{x})}
$$

We have

$$
\nabla \alpha_{i}(\mathbf{x})=\frac{f_{i}}{d_{i}}\left(\mathbf{c}_{i}-\mathbf{x}\right)
$$

where $f_{i}$ is the Lebesgue measure (length in 2D, area in 3D) of the Voronoi facet between $n_{i}$ and $\mathbf{x}, d_{i}$ is the distance between $n_{i}$ and $\mathbf{x}$, and $\mathbf{c}_{i}$ is the vector containing the co-ordinates of the centroid of the Voronoi facet between $n_{i}$ and $\mathbf{x}$. If we consider the 2D case of Figure 2, the Voronoi facet between node $n_{1}$ and $\mathbf{x}$ is the segment $a-e$. The Sibson shape functions derivatives are then expressed by

$$
\nabla \phi_{i}(\mathbf{x})=\frac{\nabla \alpha_{i}\left(\sum_{j=1}^{n} \alpha_{j}(\mathbf{x})\right)-\alpha_{i}(\mathbf{x})\left(\sum_{j=1}^{n} \nabla \alpha_{j}\right)}{\left(\sum_{j=1}^{n} \alpha_{j}(\mathbf{x})\right)^{2}}
$$

\section{Remark}

The main difference between the reproducing-b-NEM and the b-NEM without additional reproducing properties is that physical co-ordinates must be associated with each $\mathrm{K}-\mathrm{S}$ shape function, in order to evaluate the terms $p_{j}\left(\mathbf{x}_{i}\right)$ and $p_{k}\left(\mathbf{x}_{i}\right)$ in Equations (12) and (13). A simple solution is to consider the $\mathrm{K}-\mathrm{S}$ centroid co-ordinates.

In the following, the b1-NEM and b2-NEM schemes described in the previous section are corrected using the MLS procedure just described. In the most unfavourable case a point $\mathbf{x}$ is influenced by four shape functions in the b1-NEM (3 NEM shape functions, and 1 bubble shape function associated with the Delaunay triangle), and being these weight functions independent, the method is stable if the basis $\mathbf{p}^{\mathrm{T}}(\mathbf{x})$ contains 4 monomials. We call b1-NEM ${ }^{+}$the enrichment of the b1-NEM from $\mathbf{p}^{\mathrm{T}}(\mathbf{x})=\{1, x, y, x y\}$. Following similar assumptions, $\mathrm{b}^{2}-\mathrm{NEM}^{+}$results from the enrichment of the b2-NEM using $\mathbf{p}^{\mathrm{T}}(\mathbf{x})=\left\{1, x, y, x y, x^{2}, y^{2}\right\}$.

\subsection{Boundary conditions}

3.3.1. Essential boundary conditions. In the b-NEM, the essential boundary conditions can be enforced directly, as in the standard NEM, due to the nullity of internal bubble functions on the domain boundary, which we prove in the following paragraph. 
Property 1: Nullity of internal bubble functions along the boundary.

\section{Proposition}

Let $\phi_{i}^{*}(\mathbf{x})$ an internal bubble function such as:

(a) $\phi_{i}^{*}(\mathbf{x})$ is associated with a Delaunay triangle in $\Omega$; or

(b) $\phi_{i}^{*}(\mathbf{x})$ is associated with a Delaunay edge $E^{\prime} \notin \Gamma$.

Then $\phi_{i}^{*}(\mathbf{x})$ vanishes over the convex boundary $\Gamma$ of the domain.

\section{Proof}

It has been shown in Reference [12] that influence of interior nodes vanishes along the convex boundaries of the domain, due to the infinite Lebesgue measure of the second-order Voronoi cells used in the computation of the natural neighbour shape functions (see Figure 2(b)). If we consider a point $\mathbf{x}$ lying over a segment formed by two nodes, $n_{1}$ and $n_{2}$, of the boundary (see Figure 4), we have

$$
\phi_{1}(\mathbf{x}) \neq 0, \quad \phi_{2}(\mathbf{x}) \neq 0, \quad \phi_{3}(\mathbf{x})=0
$$

In the context of the b1-NEM (one bubble function associated with each Delaunay triangle) we have (see Figure 4(a)):

$$
\phi_{4}^{*}(\mathbf{x})=\phi_{1}(\mathbf{x}) \phi_{2}(\mathbf{x}) \phi_{3}(\mathbf{x})
$$

with $\phi_{i}(\mathbf{x})$ the NEM shape function associated with the node $n_{i}$ computed at point $\mathbf{x}$. As $\mathbf{x}$ reaches the boundary $\Gamma, \phi_{3}(\mathbf{x})$ vanishes, implying the nullity of $\phi_{4}^{*}(\mathbf{x})$ (see Figure 5).In the context of the b2-NEM (one bubble function associated with each Delaunay edge), we have, $\mathbf{x}$ lying over $\Gamma$ (see Figure 4(b)):

$$
\phi_{4}^{*}(\mathbf{x})=\phi_{1}(\mathbf{x}) \phi_{2}(\mathbf{x}) \neq 0, \quad \phi_{5}^{*}(\mathbf{x})=\phi_{1}(\mathbf{x}) \phi_{3}(\mathbf{x})=0, \quad \phi_{6}^{*}(\mathbf{x})=\phi_{2}(\mathbf{x}) \phi_{3}(\mathbf{x})=0
$$

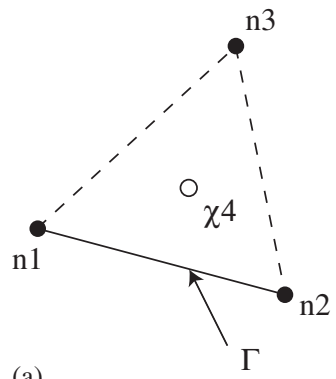

(a)

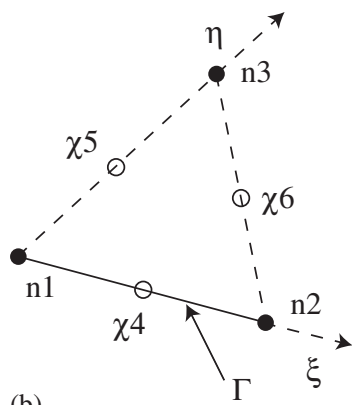

(b)

Figure 4. Nullity of internal shape functions over convex boundaries (filled circles refer to the original nodes and the empty ones are not real nodes, they indicate the existence of a bubble shape function related to the geometrical entity where the circle is located). 


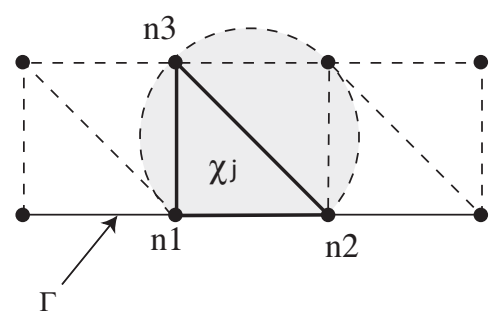

(a)

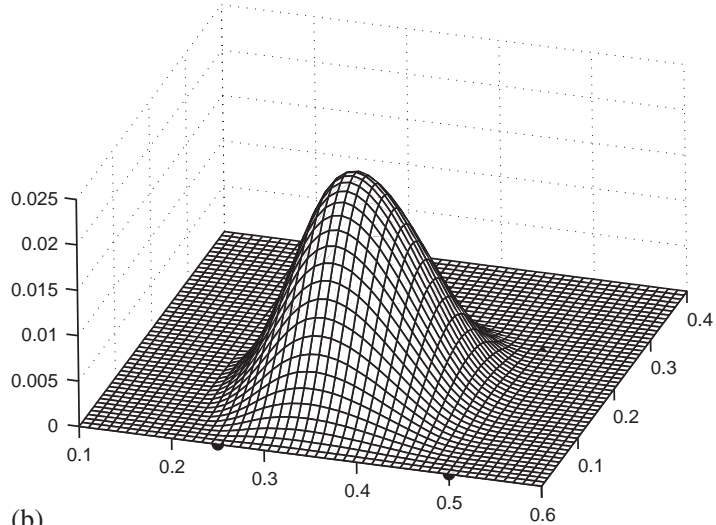

(b)

Figure 5. (a) Support of the bubble shape function of $\chi_{j}$; and (b) plot of the bubble shape function of $\chi_{j}$ (triangle $n_{1}, n_{2}, n_{3}$ ).

\section{Remark}

Property 1 is true over any kind of boundary, convex or not, if a criterion is introduced to restrict influences between nodes over non-convex boundaries, e.g. a visibility criterion (C-NEM approach [21]) or the $\alpha$-shape formalism ( $\alpha$-NEM approach [22]). In the following, we assume that such criterion is used to generalize all the results to any kind of boundary, convex or not.

Now, we prove how this property allows direct enforcement of essential boundary conditions.

- b1-NEM

In the context of the b1-NEM, all the bubble functions vanish over the boundaries $\Gamma$. Due to the Kronecker delta property and the strict linearity [12] of the NEM shape functions over the boundaries, essential boundary conditions can be enforced directly. This is also true for corrected shape functions $\left(\mathrm{b} 1-\mathrm{NEM}^{+}\right)$.

- b2-NEM

Let $\mathbf{x}$ be a point lying over the boundary $\Gamma$ (see Figure $4(\mathrm{~b})$ ). Due to property 1, we have, in the context of the b2-NEM:

$$
u^{h}(\mathbf{x})=\phi_{1}(\mathbf{x}) u_{1}+\phi_{2}(\mathbf{x}) u_{2}+\phi_{1}(\mathbf{x}) \phi_{2}(\mathbf{x}) \gamma
$$

where $\phi_{i}(\mathbf{x})$ is the linear shape function related to node $n_{i} \in \Gamma, x \in \Gamma, u_{i}$ is the nodal value at node $n_{i}$, and $\gamma$ an additional degree of freedom related to the bubble shape function. As the bubble function $\phi_{1}(\mathbf{x}) \phi_{2}(\mathbf{x})$ vanishes at the nodes, we have $u^{h}\left(\mathbf{x}_{i}\right)=u_{i}$. The exact values of the field $u^{\mathrm{ex}}\left(\mathbf{x}_{i}\right)$ can thus be enforced at the nodes $n_{i} \in \Gamma$. Using Equation (25), the value of $\gamma$ is set according to

$$
\gamma=\frac{u^{\mathrm{ex}}(\overline{\mathbf{x}})-\left(\phi_{1}(\overline{\mathbf{x}}) u^{\mathrm{ex}}\left(\mathbf{x}_{1}\right)+\phi_{2}(\overline{\mathbf{x}}) u^{\mathrm{ex}}\left(\mathbf{x}_{2}\right)\right)}{\phi_{1}(\overline{\mathbf{x}}) \phi_{2}(\overline{\mathbf{x}})}
$$

where $\overline{\mathbf{x}}$ is an arbitrary position along the edge $n_{1}-n_{2}$ (i.e. the middle of the edge). In this form we can enforce exactly linear or quadratic essential boundary conditions. 


\section{- b2-NEM ${ }^{+}$}

In the context of the $\mathrm{b} 2-\mathrm{NEM}^{+}$, we firstly prove that the resulting shape functions match exactly the 1D quadratic FE shape functions along the boundary edge $n_{1}-n_{2}$, allowing direct imposition of the essential boundary conditions over the boundary, being exact for linear or quadratic essential boundary conditions. For this purpose, we introduce the following property:

Property 2: Quadratic b2-NEM ${ }^{+}$shape function along convex boundaries.

As discussed in Section 3.1, physical co-ordinates must be associated with each K-S in the context of the reproducing b-NEM. Let $n\left(\chi_{j}\right)$ the centroid of an edge $\chi_{j}$ generated by two nodes.

\section{Proposition}

The b2-NEM ${ }^{+}$shape functions associated with the nodes $n_{l} \in S, n_{l} \in \Gamma$, and with nodes $n\left(\chi_{j}\right)$, $\chi_{j} \in \Gamma$ match the 1D quadratic Lagrange FE shape functions.

\section{Proof}

We consider, in the situation depicted in Figure 6, a point located on an edge $\chi_{3} \in \Gamma, E^{\prime}$ being generated by the nodes $n_{1}-n_{2}$. Let $n\left(\chi_{3}\right)$ the node associated with the edge $\chi_{3}$. The position of point $\xi$ is expressed in the $1 \mathrm{D}$ basis $\left(\mathbf{n}_{1}, \xi\right)$, with $\xi=\mathbf{n}_{\mathbf{1}} \mathbf{n}_{\mathbf{2}} /\left\|\mathbf{n}_{\mathbf{1}} \mathbf{n}_{\mathbf{2}}\right\|$. We set $\xi_{1}=0, \xi_{2}=1$ and $\xi_{3}=1 / 2$ (see Figure 6(a)). As influence of interior nodes vanish, the shape functions associated with nodes $n_{1}$ and $n_{2}$ become linear. The weight functions $w_{i}(\mathbf{x})$ used to compute $A(\xi)$ and $B(\xi)$ in Equations (12) and (13) are then given by

$$
w_{1}(\xi)=1-\xi, \quad w_{2}(\xi)=\xi, \quad w_{3}(\xi)=\xi(1-\xi)
$$

Introducing the weight functions (27) into (12) and (13), we obtain the following closed form for matrix $\mathbf{A}(\xi)$ and $\mathbf{B}(\xi)$, with $\mathbf{p}(\xi)^{\mathrm{T}}=\left[1, \xi, \xi^{2}\right]$ :

$$
\begin{aligned}
& \mathbf{A}(\xi)=\left\{\begin{array}{lll}
\left(1+\xi-\xi^{2}\right) & \frac{\xi}{2}(3-\xi) & \frac{\xi}{4}(5-\xi) \\
\frac{\xi}{2}(3-\xi) & \frac{\xi}{4}(5-\xi) & \frac{\xi}{8}(9-\xi) \\
\frac{\xi}{4}(5-\xi) & \frac{\xi}{8}(9-\xi) & \frac{\xi}{16}(17-\xi)
\end{array}\right\} \\
& \mathbf{B}(\xi)=\left\{\begin{array}{lll}
(1-\xi) & \xi & \xi(1-\xi) \\
0 & \xi & \frac{\xi}{2}(1-\xi) \\
0 & \xi & \frac{\xi}{4}(1-\xi)
\end{array}\right\}
\end{aligned}
$$




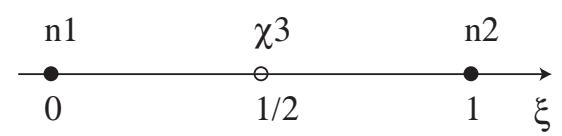

(a)

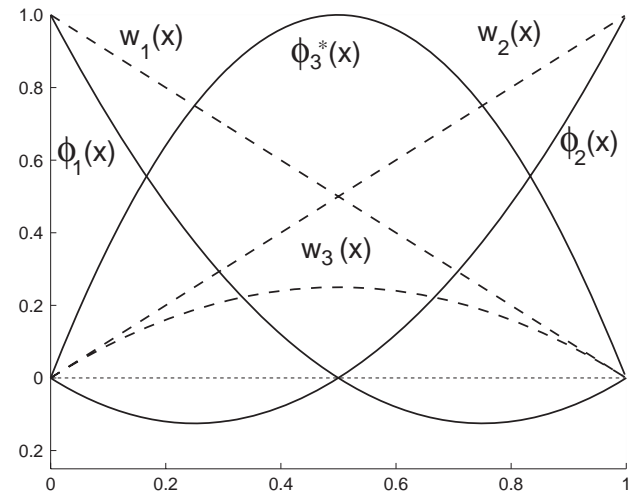

(b)

$\xi$

Figure 6. One-dimensional b2-NEM ${ }^{+}$weight and shape functions.

Applying the standard procedure, we obtain the following closed form for $A(\xi)^{-1}$ :

$$
\mathbf{A}(\xi)^{-1}=\left\{\begin{array}{ccc}
\frac{1}{(1-\xi)} & \frac{3}{(\xi-1)} & \frac{2}{(1-\xi)} \\
\frac{3}{(\xi-1)} & \frac{8 \xi+17}{\xi(1-\xi)} & \frac{2(2 \xi+9)}{\xi(\xi-1)} \\
\frac{2}{(1-\xi)} & \frac{2(2 \xi+9)}{\xi(\xi-1)} & \frac{20}{\xi(1-\xi)}
\end{array}\right\}
$$

It thus leads to

$$
\mathbf{N}(\xi)=p^{\mathrm{T}}(\xi) \mathbf{A}(\xi)^{-1} \mathbf{B}(\xi)
$$

whose components result in

$$
\begin{aligned}
& N_{1}(\xi)=\phi_{1}(\xi)=1-3 \xi+2 \xi^{2} \\
& N_{2}(\xi)=\phi_{2}(\xi)=\xi(2 \xi-1) \\
& N_{3}(\xi)=\phi_{3}^{*}(\xi)=4 \xi(1-\xi)
\end{aligned}
$$

which precisely match the 1D Lagrange polynomial shape function of second degree:

$$
N_{i}(\xi)=\frac{\left(\xi-\xi_{j}\right)\left(\xi-\xi_{k}\right)}{\left(\xi_{i}-\xi_{j}\right)\left(\xi_{i}-\xi_{k}\right)} \quad 1 \leqslant i \leqslant 3, \quad i \neq j \neq k
$$

by setting $\xi_{1}=0, \xi_{2}=1, \xi_{3}=1 / 2$. The b2-NEM ${ }^{+}$weight and shape function along a boundary are depicted in Figure 6(b).

We thus have $u^{h}\left(\mathbf{x}_{i}\right)=u_{i}$ and $u^{h}\left(\mathbf{x}_{j}\right)=\gamma_{j}, \mathbf{x}_{j}$ being the centroid of the Delaunay edge of the boundary $\Gamma$. In this context, the additional degrees of freedom associated with the edges 


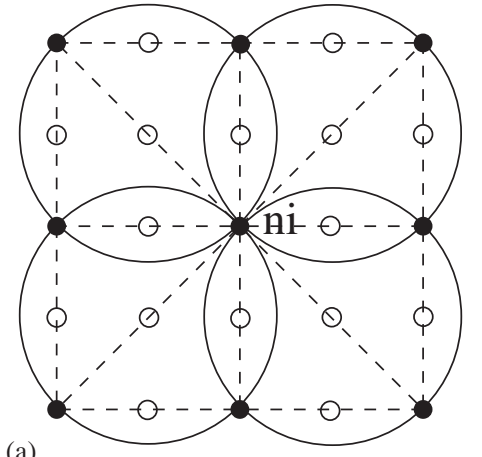

(a)

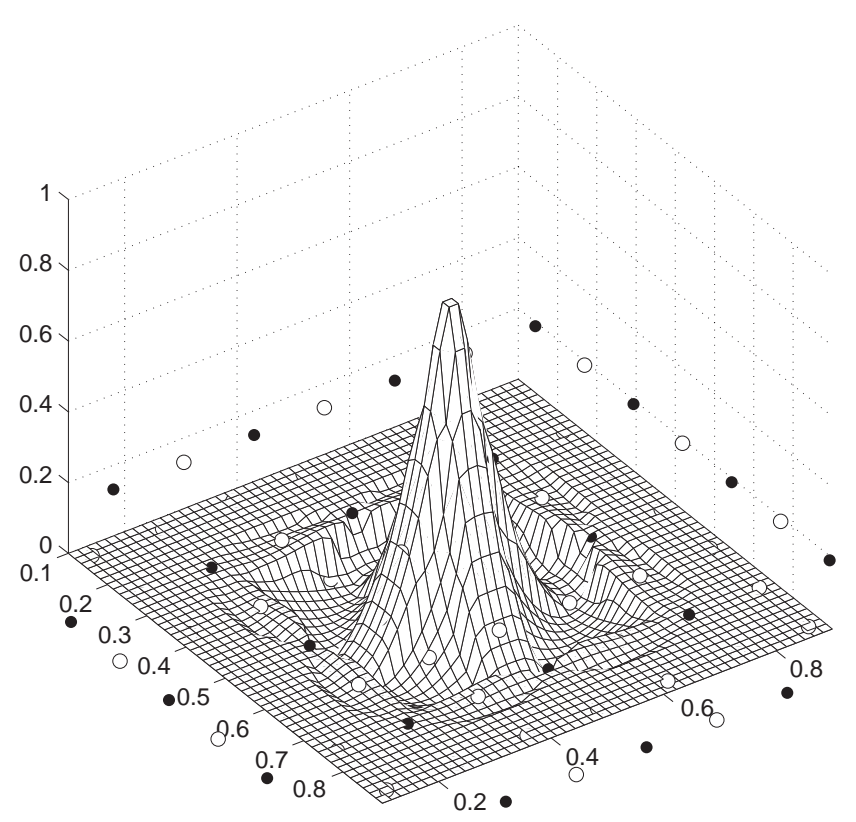

(c)

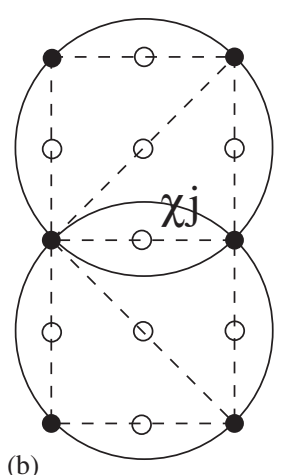

(b)

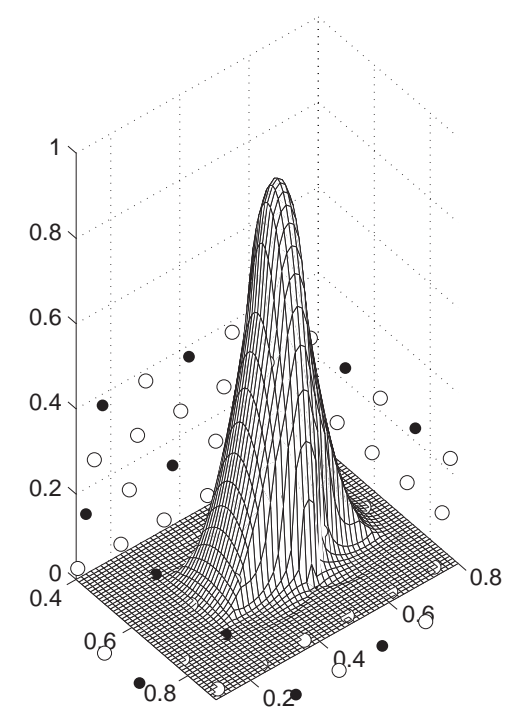

(d)

Figure 7. (a) Shape function support of $n_{i}$; (b) shape function support of $\chi_{j}$; (c) shape function related to $n_{i}$; and (d) shape function related to $\chi_{j}$.

of the boundary $\Gamma$ can be enforced by imposing the value of the exact field $u^{\text {ex }}\left(\mathbf{x}_{j}\right)$. Some plots of b2-NEM ${ }^{+}$shape functions in the domain are depicted in Figure 7.

3.3.2. Natural boundary conditions. As the b1-NEM shape functions associated with the additional degrees of freedom vanish along the boundaries, and as the b2-NEM shape functions can be expressed analytically along the boundaries as linear or quadratic polynomials, the boundary integral related to the natural boundary conditions can be easily evaluated. 


\section{PROBLEM FORMULATION}

\subsection{Strong form}

In order to analyse the just proposed approximations, we consider the mixed displacement/ pressure formulation in linear elastostatics.

Consider an open bounded domain $\Omega \in \mathfrak{R}^{2}$ with boundary $\Gamma$, being $\bar{\Omega}=\Omega \cup \Gamma$ the closure of $\Omega$. Let $\mathbf{n}$ be the unit outwards vector defined on $\Gamma$. Here we consider both the compressible and the incompressible material behaviour. Traction is prescribed on the boundary $\Gamma_{t}$ being the displacement imposed in the complementary part of the boundary $\Gamma_{u}(\bar{\Omega}=\Omega \cup \Gamma)$. Let $\boldsymbol{\sigma}$ be the Cauchy stress tensor, $\mathbf{u}$ the displacement field, and $\mathbf{b}$ the body force. The elastostatic boundary value problem is given by

$$
\begin{aligned}
& \boldsymbol{\nabla} \cdot \boldsymbol{\sigma}+\mathbf{b}=\mathbf{0} \text { in } \Omega \\
& \boldsymbol{\nabla} \cdot \mathbf{u}+\frac{p}{\lambda}=0 \quad \text { in } \Omega \\
& \mathbf{u}=\overline{\mathbf{u}} \quad \text { on } \Gamma_{u} \\
& \boldsymbol{\sigma} \cdot \mathbf{n}=\overline{\mathbf{t}} \quad \text { on } \Gamma_{t}
\end{aligned}
$$

where the Cauchy stress tensor $\boldsymbol{\sigma}$ is related to the strain tensor $\boldsymbol{\varepsilon}$ in isotropic linear elastostatics from the constitutive equation:

$$
\boldsymbol{\sigma}=-p \mathbf{1}+2 \mu \boldsymbol{\varepsilon}
$$

In Equation (38), $\lambda$ and $\mu$ are the Lamé coefficients which for plane strain are defined as

$$
\lambda=\frac{v E}{(1+v)(1-2 v)}, \quad \mu=\frac{E}{2(1+v)}
$$

where $E$ is the Young's modulus and $v$ is the Poisson's coefficient. The kinematical relation between the linearized strain tensor $\boldsymbol{\varepsilon}$ and the displacement vector $\mathbf{u}$ is

$$
\varepsilon=\frac{1}{2}\left(\nabla \mathbf{u}+(\nabla \mathbf{u})^{\mathrm{T}}\right)
$$

\subsection{Weak form}

We first define the trial and test functional spaces for the displacements and the pressure approximation. Following Hughes [36] we let $\mathbf{u} \in \mathbf{V}=\left(H^{1}(\Omega)\right)^{2}, \delta \mathbf{u} \in \mathbf{V}_{0}=\left(H_{0}^{1}(\Omega)\right)^{2}, p, \delta p \in$ $\mathbf{P}=L^{2}(\Omega)$, being $H^{1}(\Omega)$ and $H_{0}^{1}(\Omega)$ the usual Sobolev functional spaces and $L^{2}(\Omega)$ the Lebesgue space. In the numerical implementation, finite-dimensional subspaces are used as the trial and test spaces for the displacements and pressure. Let $\mathbf{V}^{h} \subset \mathbf{V}$ and $\mathbf{V}_{0}^{h} \subset \mathbf{V}_{0}$ be the trial and test spaces for the displacements, and $\mathbf{P}^{h} \subset \mathbf{P}$ be the trial and test space for the pressure. Since the test functions $\delta \mathbf{u}^{h}$ and $\delta p^{h}$ are independent and arbitrary, we obtain the Hermann variational form associated with Equations (34) and (35): 
Find $\left(\mathbf{u}^{h}, p^{h}\right) \in \mathbf{V}^{h} \times \mathbf{P}^{h}$ such that

$$
\begin{aligned}
& \mathbf{a}\left(\mathbf{u}^{h}, \delta \mathbf{u}^{h}\right)+b\left(\delta \mathbf{u}^{h}, p^{h}\right)=\int_{\Omega} \mathbf{b} \cdot \delta \mathbf{u} \mathrm{d} \Omega+\int_{\Gamma_{u}} \overline{\mathbf{t}} \cdot \delta \mathbf{u} \mathrm{d} \Gamma \quad \forall \delta \mathbf{u}^{h} \in \mathbf{V}_{0}^{h} \\
& b\left(\mathbf{u}^{h}, \delta p^{h}\right)-\frac{1}{\lambda}\left(p^{h}, \delta p^{h}\right)=0 \quad \forall \delta p^{h} \in \mathbf{P}^{h}
\end{aligned}
$$

where

$$
\begin{aligned}
& a\left(\mathbf{u}^{h}, \delta \mathbf{u}^{h}\right)=2 \mu \int_{\Omega} \boldsymbol{\varepsilon}\left(\mathbf{u}^{h}\right): \boldsymbol{\varepsilon}\left(\delta \mathbf{u}^{h}\right) \mathrm{d} \Omega \\
& b\left(\delta \mathbf{u}^{h}, p^{h}\right)=-\int_{\Omega} p^{h} \nabla \cdot \delta \mathbf{u}^{h} \mathrm{~d} \Omega
\end{aligned}
$$

In a displacement/pressure mixed formulation for linear elasticity, the bilinear form $a\left(\mathbf{u}^{h}, \delta \mathbf{u}\right)$ : $V^{h} \times V_{0} \rightarrow \Re$ is symmetric, bounded, and positive definite. Hence, for the displacement-pressure pair $(\mathbf{u}, p)$ to be the unique solution in Equation (41), the bilinear forms $a\left(\mathbf{u}^{h}, \delta \mathbf{u}\right)$ and $b\left(\delta \mathbf{u}^{h}, p^{h}\right): V_{0} \times P \rightarrow \Re$ must satisfy the following conditions [4]: (i) the continuity of $a(.,$. and $b(.,$.$) , (ii) the coercivity of a(.,$.$) and b(.,$.$) , and (iii) b(.,$.$) must satisfy the LBB$ condition:

$$
\inf _{p^{h} \in P^{h}} \sup _{v^{h} \in V^{h}} \frac{\left|b\left(\mathbf{u}^{h}, p^{h}\right)\right|}{\left\|\mathbf{u}^{h}\right\|_{V}\left\|p^{h}\right\|_{P}} \geqslant \beta>0
$$

\subsection{Natural element discretization}

We consider a Galerkin procedure where displacement trial and test functions are interpolated using the same shape functions, as the same for the pressure trial and test functions. In the following, the pressure is interpolated using the standard (Sibson) NEM shape functions, while the displacements are interpolated using the b-NEM shape functions defined in Section 3.

4.3.1. b-NEM displacements interpolation. In the context of the b-NEM, the following approximation scheme is used for the displacements interpolation:

$$
\begin{aligned}
& \mathbf{u}^{h}(\mathbf{x})=\sum_{i=1}^{n} \phi_{i}(\mathbf{x}) \mathbf{u}_{i}+\sum_{j=1}^{m} \phi_{j}^{*}(\mathbf{x}) \gamma_{j}, \quad \delta \mathbf{u}^{h}(\mathbf{x})=\sum_{i=1}^{n} \phi_{i}(\mathbf{x}) \delta \mathbf{u}_{i}+\sum_{j=1}^{m} \phi_{j}^{*}(\mathbf{x}) \delta \gamma_{j} \\
& p^{h}(\mathbf{x})=\sum_{i=1}^{n} \phi_{i}(\mathbf{x}) p_{i}, \quad \delta p(\mathbf{x})=\sum_{i=1}^{n} \phi_{i}(\mathbf{x}) \delta p_{i}
\end{aligned}
$$

where $\phi_{i}(\mathbf{x})$ is the usual (Sibson) NEM shape function related to node $n_{i}$ computed at point $\mathbf{x}$, $\phi_{j}^{*}(\mathbf{x})$ is the bubble shape function associated with the K-S $\chi_{j}$, being $\gamma_{j}$ the degree of freedom associated with $\chi_{j}, n$ the number of neighbour nodes related to point $\mathbf{x}$ and $m$ the number of influent K-S at point $\mathbf{x}$ (number of K-S shape functions whose support contains $\mathbf{x}$ ). The strain-displacement relation for the displacement trial function can be written as

$$
\boldsymbol{\varepsilon}^{h}(\mathbf{x})=\left\{\mathbf{B}\left(\phi_{i}\right) ; \mathbf{B}\left(\phi_{i}^{*}\right)\right\}\left[\begin{array}{l}
\mathbf{u} \\
\gamma
\end{array}\right]
$$


where

$$
\begin{aligned}
\mathbf{B}\left(\phi_{i}\right) & =\left[\begin{array}{ccccccc}
\phi_{1, X} & 0 & \phi_{2, X} & 0 & \ldots & \phi_{N, X} & 0 \\
0 & \phi_{1, Y} & 0 & \phi_{2, Y} & \ldots & 0 & \phi_{N, Y} \\
\phi_{1, Y} & \phi_{1, X} & \phi_{2, Y} & \phi_{2, X} & \ldots & \phi_{N, Y} & \phi_{N, X}
\end{array}\right] \\
\mathbf{B}\left(\phi_{i}^{*}\right)= & {\left[\begin{array}{ccccccc}
\phi_{1, X}^{*} & 0 & \phi_{2, X}^{*} & 0 & \ldots & \phi_{M, X}^{*} & 0 \\
0 & \phi_{1, Y}^{*} & 0 & \phi_{2, Y}^{*} & \ldots & 0 & \phi_{M, Y}^{*} \\
\phi_{1, Y}^{*} & \phi_{1, X}^{*} & \phi_{2, Y}^{*} & \phi_{2, X}^{*} & \ldots & \phi_{M, Y}^{*} & \phi_{M, X}^{*}
\end{array}\right] }
\end{aligned}
$$

where $N$ are the total number of original nodes and $M$ the number of $\mathrm{K}-\mathrm{S}$ shape functions considered.

The divergence of the displacement trial solution is given by

$$
\nabla \cdot \mathbf{u}^{h}(\mathbf{x})=\left\{\tilde{\mathbf{B}}\left(\phi_{i}\right) ; \tilde{\mathbf{B}}\left(\phi_{i}^{*}\right)\right\}\left[\begin{array}{l}
\mathbf{u}_{i} \\
\gamma
\end{array}\right]
$$

where

$$
\begin{aligned}
\tilde{\mathbf{B}}\left(\phi_{i}\right) & =\left[\begin{array}{lllllll}
\phi_{1, X} & \phi_{1, Y} & \phi_{2, X} & \phi_{2, Y} & \ldots & \phi_{N, X} & \phi_{N, Y}
\end{array}\right] \\
\tilde{\mathbf{B}}\left(\phi_{i}^{*}\right) & =\left[\begin{array}{lllllll}
\phi_{1, X}^{*} & \phi_{1, Y}^{*} & \phi_{2, X}^{*} & \phi_{2, Y}^{*} & \ldots & \phi_{M, X}^{*} & \phi_{M, Y}^{*}
\end{array}\right]
\end{aligned}
$$

4.3.2. $b-N E M^{+}$interpolation. In the context of the $\mathrm{b}-\mathrm{NEM}^{+}$, the following approximation scheme is used for the displacements interpolation:

$$
\begin{array}{ll}
\mathbf{u}^{h}(\mathbf{x})=\sum_{i=1}^{n+m} \psi_{i}(\mathbf{x}) \mathbf{u}_{i}, & \delta u^{h}(\mathbf{x})=\sum_{i=1}^{n+m} \psi_{i}(\mathbf{x}) \delta \mathbf{u}_{i} \\
p^{h}(\mathbf{x})=\sum_{i=1}^{n} \phi_{i}(\mathbf{x}) p_{i}, & \delta p^{h}(\mathbf{x})=\sum_{i=1}^{n} \phi_{i}(\mathbf{x}) \delta p_{i}
\end{array}
$$

where $\psi_{i}(\mathbf{x})$ are the corrected shape functions computed at point $\mathbf{x}$ using the MLS technique described in Section 3.2, $n+m$ the number of influent shape functions, including nodes and K-S shape functions, $\mathbf{u}_{i}$ contains both displacements associated with the nodes, and nodes that have been associated to the K-S shape functions in the MLS procedure. Thus, it results in

$$
\boldsymbol{\varepsilon}^{h}(\mathbf{x})=\mathbf{B}\left(\psi_{i}\right) \mathbf{u}
$$

The divergence of the displacement trial solution is given by

$$
\nabla \cdot \mathbf{u}^{h}(\mathbf{x})=\tilde{\mathbf{B}}\left(\psi_{i}\right) \mathbf{u}
$$


4.3.3. Matrix form. On substituting the displacements and pressure trial and test functions in Equations (41) and (42) and using the arbitrariness of displacements and pressure variations, the following discrete system of equations is obtained [36]:

$$
\left[\begin{array}{ll}
\mathbf{K} & \mathbf{G}^{\mathrm{T}} \\
\mathbf{G} & \mathbf{M}
\end{array}\right]\left\{\begin{array}{l}
\mathbf{u} \\
\mathbf{p}
\end{array}\right\}=\left\{\begin{array}{l}
\mathbf{f} \\
\mathbf{0}
\end{array}\right\}
$$

where

$$
\begin{aligned}
\mathbf{K} & =\int_{\Omega} 2 \mu \overline{\mathbf{B}}^{\mathrm{T}} \overline{\mathbf{B}} \mathrm{d} \Omega \\
\mathbf{G} & =-\int_{\Omega} \boldsymbol{\phi}^{\mathrm{T}} \overline{\tilde{\mathbf{B}}} \mathrm{d} \Omega \\
\mathbf{M} & =-\frac{1}{\lambda} \int_{\Omega} \boldsymbol{\phi}^{\mathrm{T}} \boldsymbol{\phi} \mathrm{d} \Omega \\
\mathbf{f} & =\int_{\Omega} \mathbf{N}^{\mathrm{T}} \mathbf{b} \mathrm{d} \Omega+\int_{\Gamma_{t}} \mathbf{N}^{\mathrm{T}} \overline{\mathbf{t}} \mathrm{d} \Gamma
\end{aligned}
$$

where

$$
\begin{aligned}
&\left\{\begin{array} { l l l } 
{ \overline { \mathbf { B } } = \{ \mathbf { B } ( \phi _ { i } ) ; \mathbf { B } ( \phi _ { i } ^ { * } ) \} } & { ( \mathrm { b } - \mathrm { NEM } ) } \\
{ \overline { \mathbf { B } } = \mathbf { B } ( \psi _ { i } ) } & { ( \mathrm { b } - \mathrm { NEM } ^ { + } ) }
\end{array} \left\{\begin{array}{ll}
\overline{\tilde{\mathbf{B}}}=\left\{\tilde{\mathbf{B}}\left(\phi_{i}\right) ; \tilde{\mathbf{B}}\left(\phi_{i}^{*}\right)\right\} & (\mathrm{b}-\mathrm{NEM}) \\
\overline{\tilde{\mathbf{B}}}=\tilde{\mathbf{B}}\left(\psi_{i}\right) & \left(\mathrm{b}-\mathrm{NEM}^{+}\right)
\end{array}\right.\right. \\
& \begin{cases}\mathbf{N}=\left\{\phi_{i} ; \phi_{i}^{*}\right\} & (\mathrm{b}-\mathrm{NEM}) \\
\mathbf{N}=\psi_{i} & \left(\mathrm{~b}-\mathrm{NEM}^{+}\right)\end{cases}
\end{aligned}
$$

In the above equations, $\mathbf{u}$ is the vector containing all the displacement degrees of freedom and $\mathbf{p}$ is the vector of nodal pressures. The matrix $\mathbf{K}$ is symmetric positive definite, and the matrix $\mathbf{M}$ is symmetric $(\mathbf{M}=\mathbf{0}$ when $v=0.5)$ and the matrix $\mathbf{G}$ is the discrete divergence operator.

\subsection{The numerical inf-sup test}

The numerical inf-sup test is based in the next results:

\section{Proposition}

Let $\mathbf{M}_{\mathbf{v}}$ and $\mathbf{M}_{\mathbf{q}}$ be the mass matrices associated to the scalar product of $V_{0}^{h}$ and $P_{0}^{h}$, respectively, and let $\mu_{\min }$ be the smallest non-zero eigenvalue defined by the following eigenproblem:

$$
\mathbf{G}^{\mathrm{T}} \mathbf{M}_{\mathbf{q}} \mathbf{G u}=\mu^{2} \mathbf{M}_{\mathbf{v}} \mathbf{u}
$$

Then the value of $\beta$ in Equation (45) is simply $\mu_{\min }$, and the matrix $M_{q}$ and $\mathbf{M}_{\mathbf{v}}$ defined such as:

$$
\left\|\mathbf{u}^{h}\right\|_{H^{1}}=\mathbf{u}^{h \mathrm{~T}} \mathbf{M}_{\mathbf{v}} \mathbf{u}^{h}
$$




$$
\left\|\mathbf{p}^{h}\right\|_{L^{2}}=\mathbf{p}^{h^{\mathrm{T}}} \mathbf{M}_{\mathbf{q}} \mathbf{p}^{h}
$$

and thus read

$$
\begin{aligned}
& \mathbf{M}_{\mathbf{q}}=\int_{\Omega} \boldsymbol{\phi}^{\mathrm{T}} \boldsymbol{\phi} \mathrm{d} \Omega \\
& \mathbf{M}_{\mathbf{v}}=\int_{\Omega}\left(\overline{\mathbf{B}}^{\mathrm{T}} \overline{\mathbf{B}}+\mathbf{N}^{\mathrm{T}} \mathbf{N}\right) \mathrm{d} \Omega
\end{aligned}
$$

The proof can be found in Reference [4] or [5]. The numerical test proposed in Reference [6] consists in testing a particular formulation by calculating $\beta$ using meshes of increasing refinement. On the basis of three or four results it can be predicted whether the inf-sup value is probably bounded from underneath or, on the contrary, goes down to zero when the mesh is refined. The good behaviour of this test is demonstrated on several examples of elements for the incompressible elasticity problem in Reference [6]. In the following section this test is used to check the behaviour of the proposed b-NEM mixed approximation schemes.

\section{NUMERICAL EXAMPLES}

The application of the mixed NEM to problems in small displacements and strains compressible as well as near-incompressible 2D elastostatics, in the absence of body forces, is analysed in the present section.

\subsection{Numerical test for the inf-sup condition}

In order to perform the inf-sup test a sequence of successive refined meshes is considered (uniform distributions). The objective is to monitor the inf-sup values, $\beta$, when $h$ decreases. If $\log \beta$ decreases with $\log h$, the approximation scheme does not pass the LBB numerical test, which requires that $\log \beta$ remains bounded by a positive constant when $\log h$ decreases.

Figure 8 shows numerical test comparing some mixed NEM approximation schemes, i.e. b-NEM/NEM, NEM/Thiessen [25] (NEM approximation for the displacements and constant pressure within each Voronoi cell), and the P1/P0 and P2/P1 mixed FEM approximation schemes. The FEM computations are carried out using directly the Delaunay triangles. As claimed in other previous works [26], the mixed NEM/Thiessen approximation scheme does not pass the numerical inf/sup test. The mixed FEM P1/P0 also violates the LBB condition [6]. All the bubble-NEM schemes are clearly LBB compliant, being the results similar to the ones computed by using the P2/P1 FEM, which satisfy the LBB condition.

\subsection{Cantilever beam}

Figure 9 depicts a cantilever beam subjected to a parabolic load on its left boundary. The beam has length $L$, height $D$, and unit thickness. The displacement solution can be found, for example, in Reference [37]:

$$
u=\frac{-P y}{6 \bar{E} I}\left[(6 L-3 x) x+(2+\bar{v}) y^{2}-\frac{3 D^{2}}{2}(1+\bar{v})\right]
$$




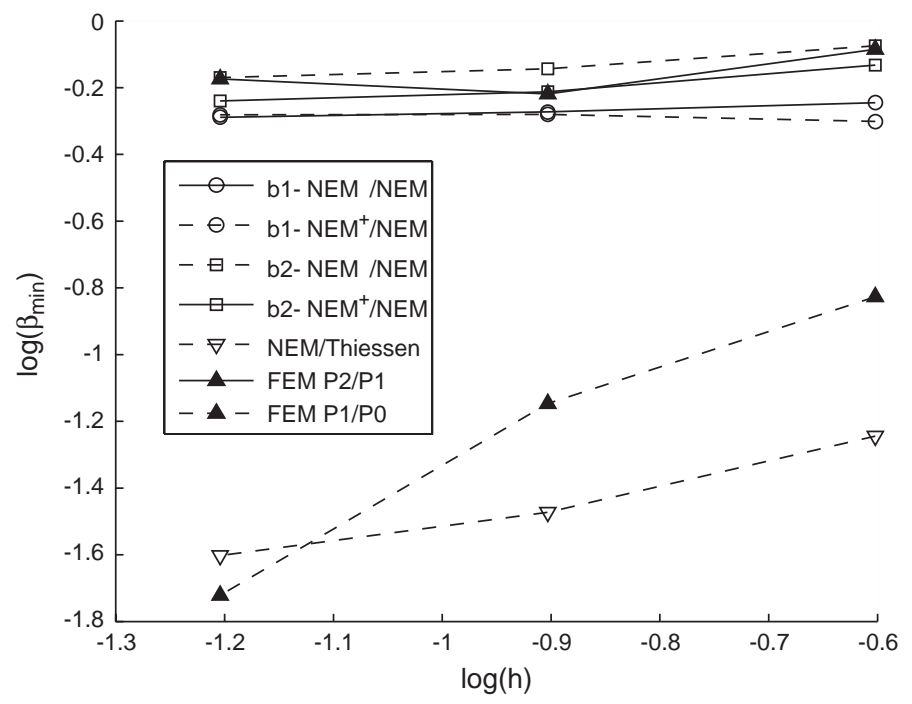

Figure 8. inf-sup numerical test.

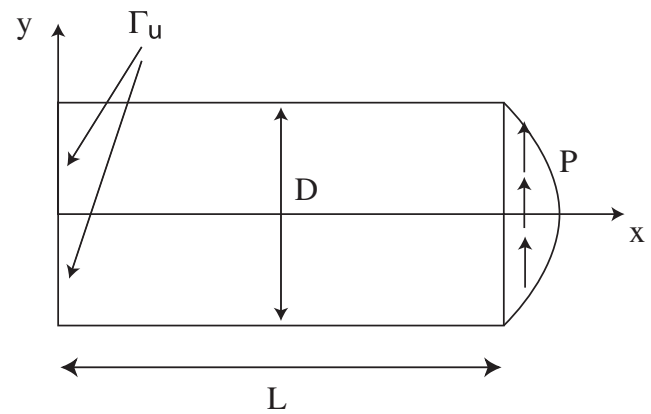

Figure 9. Geometry of the cantilever beam problem.

$$
v=\frac{P}{6 \bar{E} I}\left[3 \bar{v} y^{2}(L-x)+(3 L-x) x^{2}\right]
$$

The stress solution results in

$$
\begin{aligned}
& \sigma_{11}=\frac{-P(L-x) y}{I} \\
& \sigma_{22}=0 \\
& \sigma_{12}=\frac{P\left(\frac{D^{2}}{4}-y^{2}\right)}{2 I}
\end{aligned}
$$




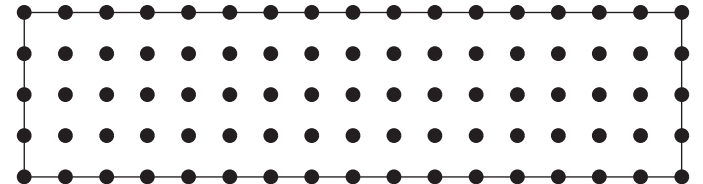

(a)

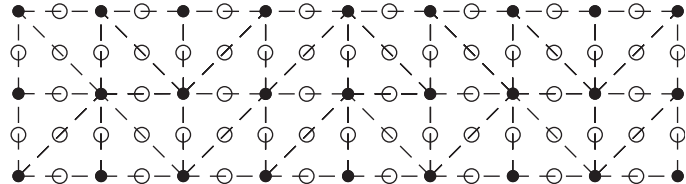

(b)

Figure 10. Nodal discretization for the cantilever beam problem, 85 nodes: (a) mixed NEM/NEM and b1-NEM/NEM; and (b) mixed b2-NEM/NEM (empty dots represent the centroid of the Delaunay edges).

The material parameters are defined in plane strain by

$$
\begin{aligned}
& \bar{E}=\frac{E}{(1+v)} \\
& \bar{v}=\frac{v}{\left(1-v^{2}\right)}
\end{aligned}
$$

and the moment of inertia $I$ related to rectangular beam cross-section with unit thickness, is:

$$
I=\frac{D^{3}}{12}
$$

In the numerical model, the analytical displacement solution (Equations (70) and (71)) is prescribed on the boundary $\Gamma_{u}: x=0,-D / 2 \leqslant y \leqslant D / 2$ (Figure 9). On the remaining part of the boundary, exact traction is enforced. The following parameters are used in the numerical computations: $P=1000 \mathrm{MPa}, D=1 \mathrm{~mm}, L=4 \mathrm{~mm}$ and plane strain conditions are assumed.

The mixed natural element displacement/pressure formulation is applied to both the compressible and near-incompressible cases. The numerical computations are carried out using different nodal discretizations, namely 85, 297 and 1105 nodes. Equal nodal spacing in the $x$ - and $y$-direction is used in each of the above nodal distributions. In the case of the b2-NEM, we consider the spacing $h$ between a node and a contiguous edge centroid. An example involving 85 nodes is shown in Figure 10. In the mixed NEM/NEM, the same set of nodes is used for both the displacements and the pressure interpolations. In the mixed b-NEM/NEM, the set of original nodes (black dot in Figure 10(b)) is used to discretize the pressure using the standard NEM interpolation scheme. The bubble shape functions are computed using Equations (7) (b-NEM) or (15) $\left(\mathrm{b}-\mathrm{NEM}^{+}\right)$. A convergence study for the cantilever beam problem is carried out, using the energy norm defined by

$$
\left\|\mathbf{u}-\mathbf{u}^{h}\right\|_{E(\Omega)}=\left(\frac{1}{2} \int_{\Omega}\left(\boldsymbol{\sigma}-\left\{-p^{h} \mathbf{1}+2 \mu \boldsymbol{\varepsilon}^{h}\right\}\right):\left(\boldsymbol{\varepsilon}-\boldsymbol{\varepsilon}^{h}\right)\right)^{1 / 2}
$$

In Figure 11 the relative energy norm is plotted against the nodal spacing $h$ on a $\log -\log$ plot. Results are presented for the NEM/NEM displacement/pressure and b1-NEM/NEM displacements/pressure formulations. The error norm computations are carried out for five different values of the Poisson's ratio: $v=0.4, v=0.49, v=0.499, v=0.49999, v=0.5-1 \times 10^{-10}$ (quasi-incompressibility). It can be noticed that a significant loss of accuracy is obtained for the NEM/NEM formulation in the incompressibility limit. On the other hand, the relative error 


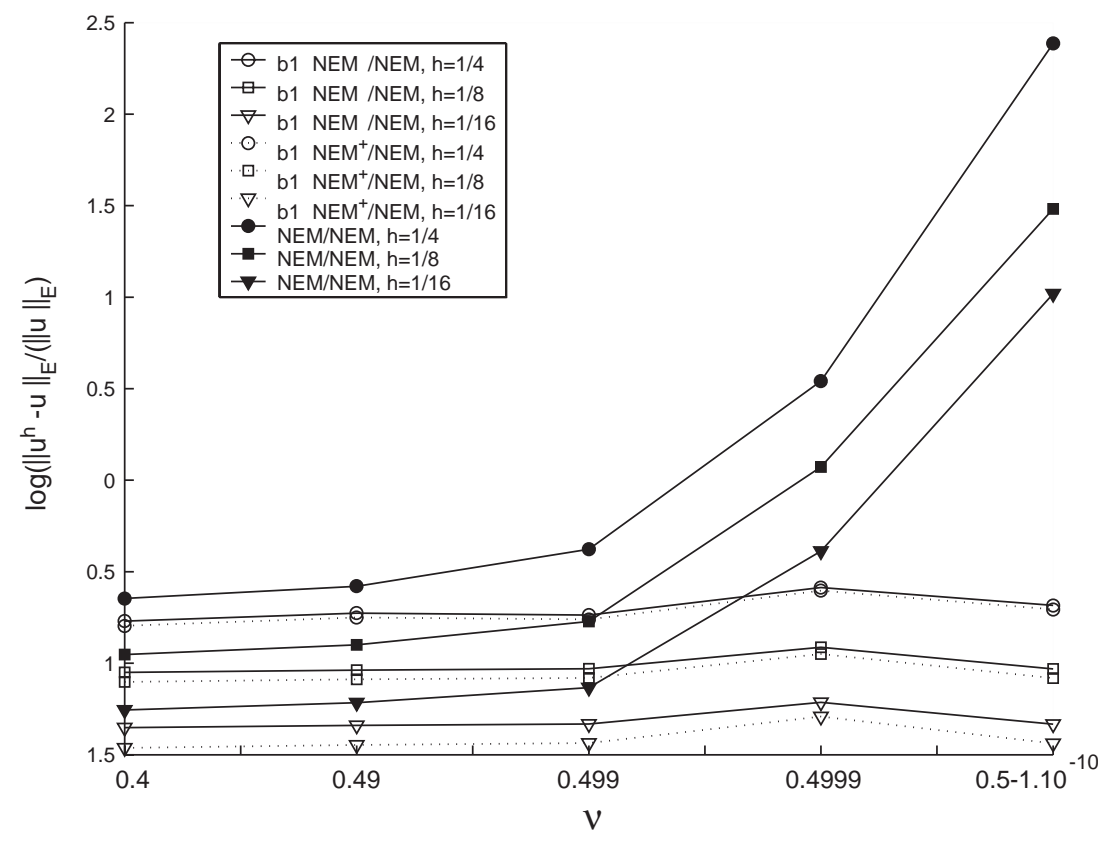

Figure 11. Error in energy norm of mixed b1-NEM/NEM approximation versus mixed NEM/NEM approximation.

norms, using the mixed b1-NEM/NEM are independent of the Poisson's ratio $v$. No significant improvement is obtained by using the MLS correction (b1-NEM ${ }^{+}$).

The same study is carried out using the mixed b2-NEM/NEM interpolation scheme. Similar results are presented in Figure 12, showing that the relative error norms using the mixed b2-NEM/NEM are independent of the Poisson's ratio $v$, even for quasi-incompressibility $\left(v=0.5-1 \times 10^{-10}\right)$. As before, no appreciable improvements are attained by using the MLS correction (b2-NEM $\left.{ }^{+}\right)$.

Finally, the convergence of the different mixed approximation schemes (b1-NEM/NEM,

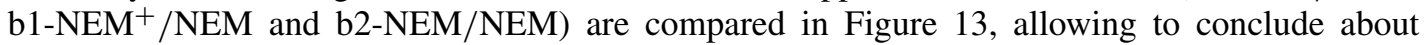
the better accuracy of mixed b2-NEM/NEM approximations.

Pressure is computed along the mid-section of the beam using the mixed b1-NEM/NEM, b2-NEM/NEM and NEM/NEM mixed approximations. In Figure 14, the variation of the pressure $p$ for the nodal spacing $h=1 / 4$ is plotted for the compressible case $(v=0.4)$. In Figures 15 and 16 the pressure is plotted for the near-incompressible case $(v=0.499), h=1 / 4$, $h=1 / 16$. The pressure oscillations for the NEM/NEM are fairly pronounced. On the contrary, the b1-NEM/NEM or the b2-NEM/NEM exhibits excellent results without any oscillations.

\subsection{Infinite plate with a circular hole}

The classical problem of an infinite plate containing a circular hole shown in Figure 17 is analysed when a unit traction in the $x$-direction is assumed far from the hole. 


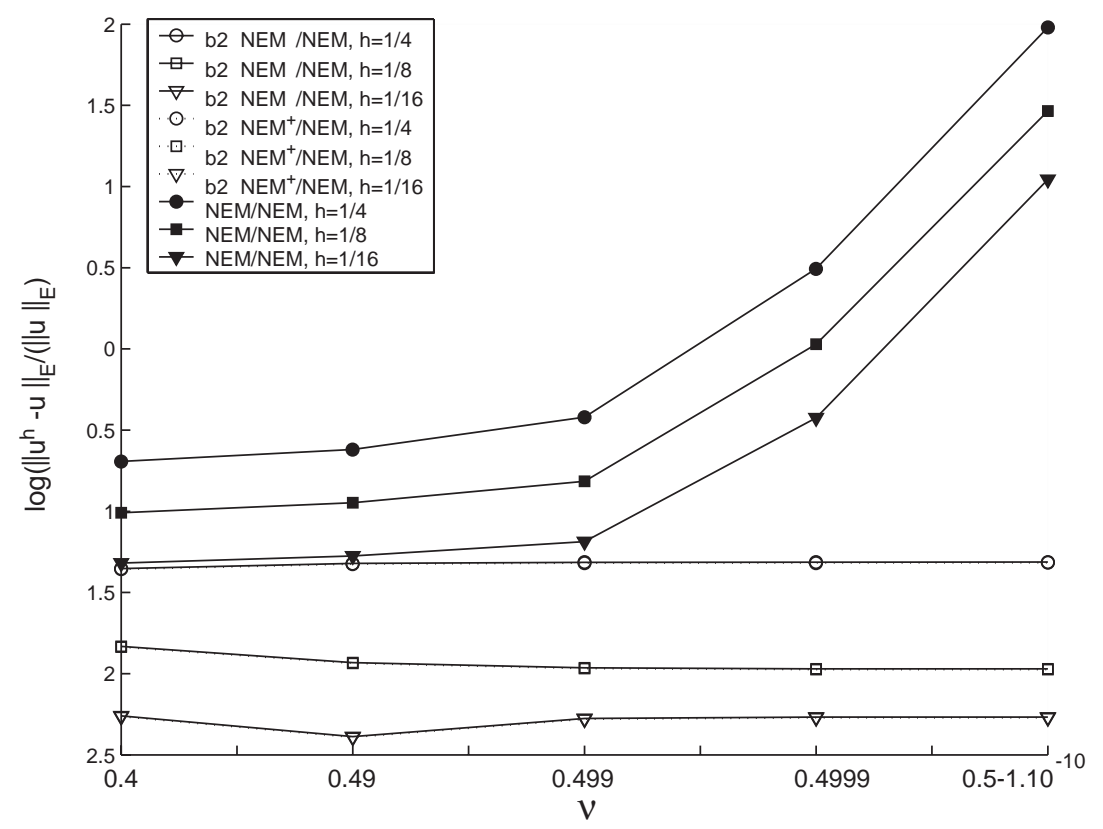

Figure 12. Error in energy norm of mixed b2-NEM/NEM approximation versus mixed NEM/NEM approximation.

The exact solution of this problem can be found in Reference [38]:

$$
\begin{aligned}
& \sigma_{11}(r, \theta)=1-\frac{a^{2}}{r^{2}}\left\{\frac{3}{2} \cos (2 \theta)+\cos (4 \theta)\right\}+\frac{3}{2} \frac{a^{4}}{r^{4}} \cos (4 \theta) \\
& \sigma_{22}(r, \theta)=-\frac{a^{2}}{r^{2}}\left\{\frac{1}{2} \cos (2 \theta)-\cos (4 \theta)\right\}-\frac{3}{2} \frac{a^{4}}{r^{4}} \cos (4 \theta) \\
& \sigma_{12}(r, \theta)=-\frac{a^{2}}{r^{2}}\left\{\frac{1}{2} \sin (2 \theta)+\sin (4 \theta)\right\}+\frac{3}{2} \frac{a^{4}}{r^{4}} \sin (4 \theta)
\end{aligned}
$$

The displacement components are then:

$$
\begin{aligned}
& u(r, \theta)=\frac{a}{8 \mu}\left[\frac{r}{a}(\kappa+1) \cos (\theta)+2 \frac{a}{r}((1+\kappa) \cos (\theta)+\cos (3 \theta))-2 \frac{a^{3}}{r^{3}} \cos (3 \theta)\right] \\
& v(r, \theta)=\frac{a}{8 \mu}\left[\frac{r}{a}(\kappa-3) \sin (\theta)+2 \frac{a}{r}((1-\kappa) \sin (\theta)+\sin (3 \theta))-2 \frac{a^{3}}{r^{3}} \sin (3 \theta)\right]
\end{aligned}
$$




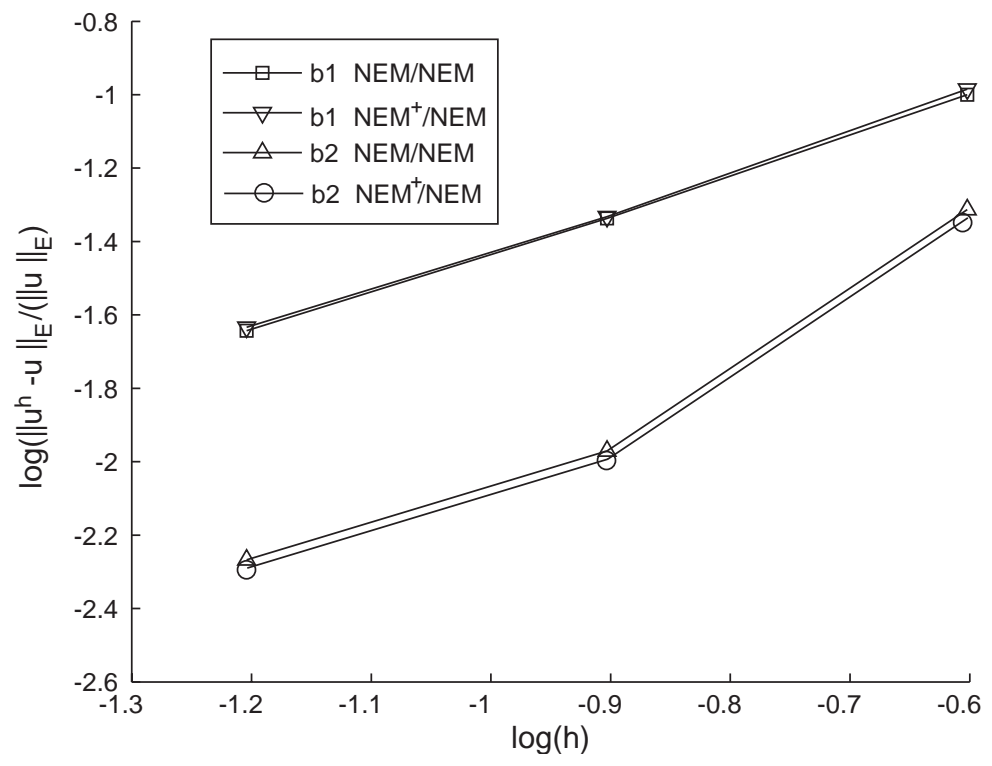

Figure 13. Error in energy norm of the different methods in the near-incompressible limit $\left(v=0.5-1 \times 10^{-10}\right)$.
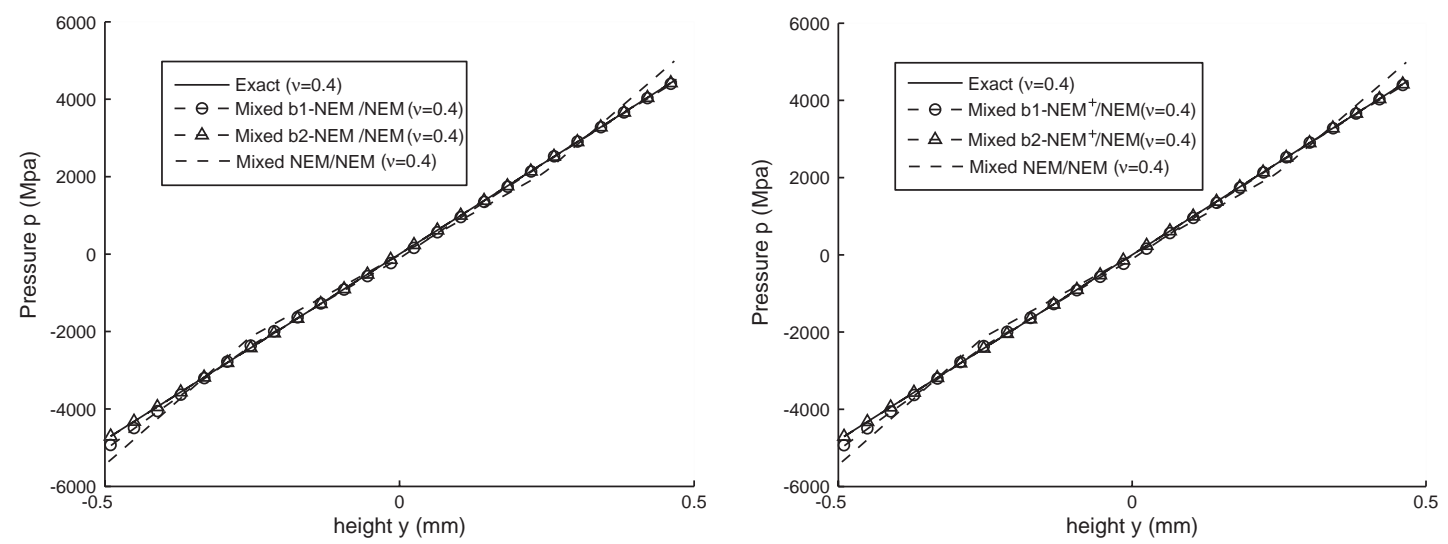

Figure 14. Pressure profile along the beam mid-section $(v=0.4, h=1 / 4)$.

where $\mu$ is the shear modulus and $\kappa$ (Kolosov constant) is defined as

$$
\kappa= \begin{cases}3-4 v & \text { (plane strain) } \\ \frac{3-v}{1+v} & \text { (plane stress) }\end{cases}
$$



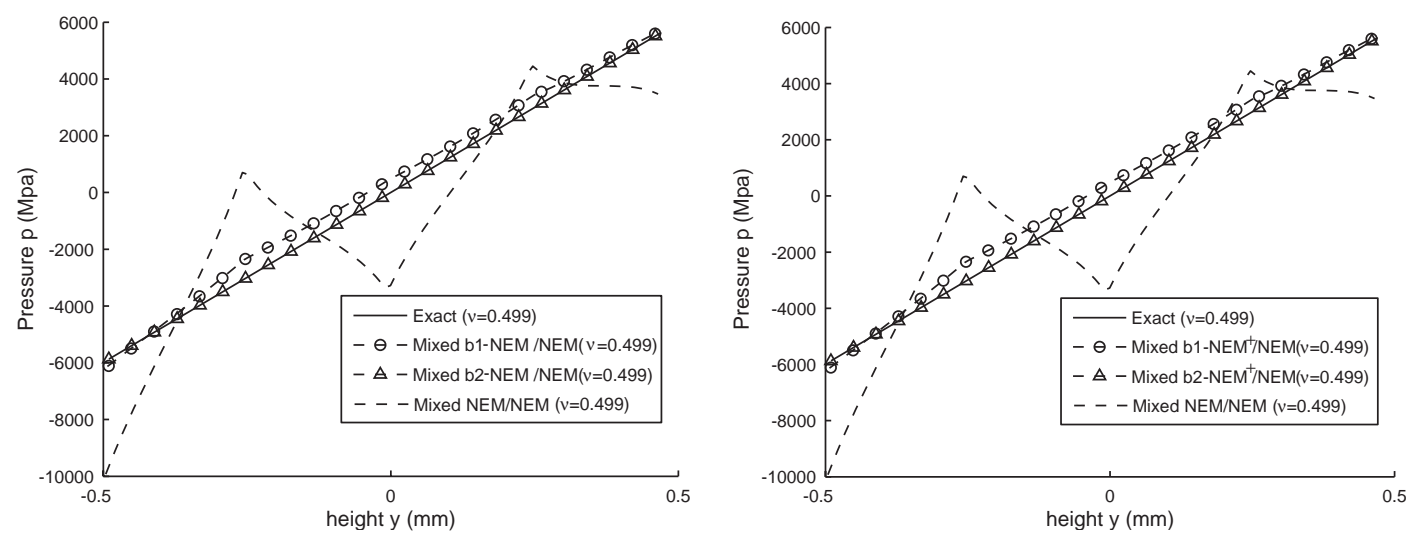

Figure 15. Pressure profile along the beam mid-section $(v=0.499, h=1 / 4)$.
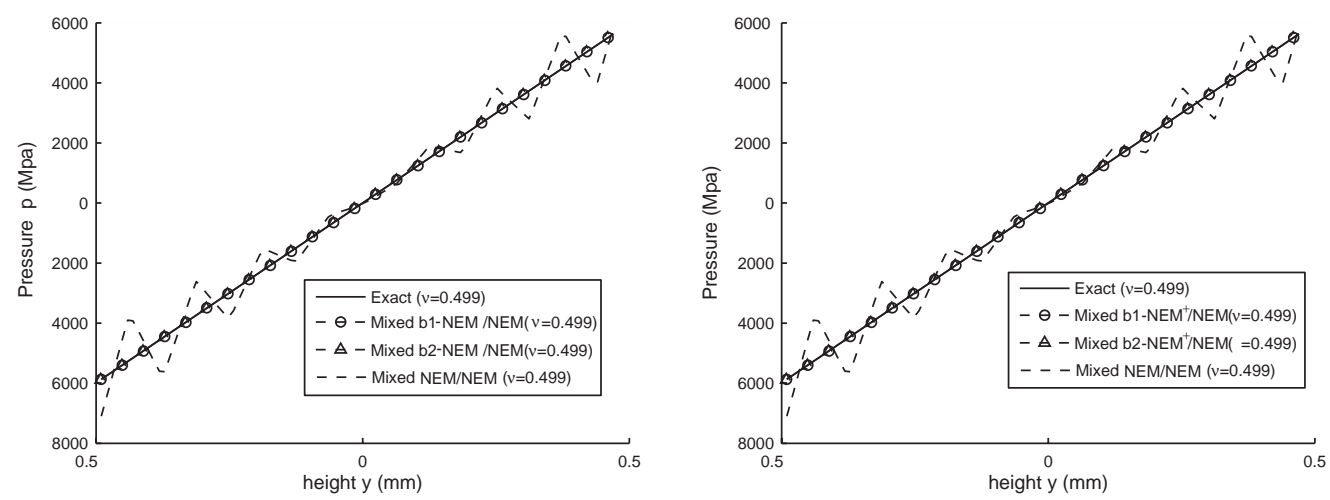

Figure 16. Pressure profile along the beam mid-section $(v=0.499, h=1 / 16)$.

The unbounded plate is truncated and modelled as a square with a central hole of radius $a=0.2 \mathrm{~mm}$ and width $2 L=2 \mathrm{~mm}$. Only one quadrant of that plate is analysed by symmetry considerations. The nodal distribution related to a spacing $h=1 / 16$ is depicted in Figure 18 .

Displacement boundary conditions are prescribed on $x=0$ and $y=0$, being the traction enforced in the remaining part of the domain boundary. Plane strain conditions are assumed.

Figure 19 compares the exact and computed pressures along $\theta=0$. The computed pressure is obtained by using the NEM/NEM, b1-NEM/NEM and b2-NEM/NEM mixed formulations when the incompressible limit is approached $(v=0.499)$. It can be noticed that the b1-NEM/NEM and b2-NEM provide excellent results and no oscillations are observed in the computed results. On the contrary, significant spurious oscillations appear when the mixed $\mathrm{NEM} / \mathrm{NEM}$ is used. 


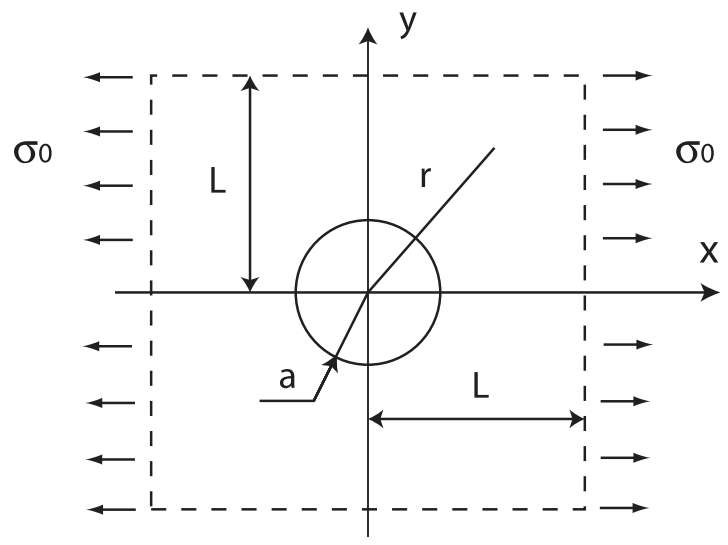

Figure 17. Plate with a circular hole under tension.

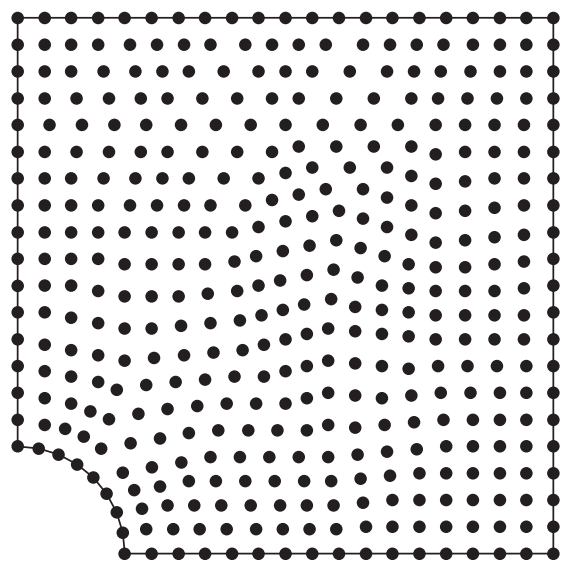

(a)

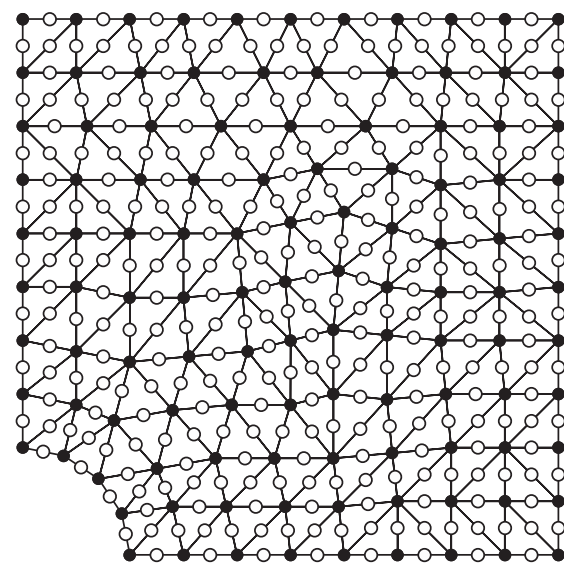

(b)

Figure 18. Nodal distribution corresponding to: (a) NEM/NEM and b1-NEM/NEM mixed formulations; and (b) b2-NEM/NEM mixed approximation.

\section{CONCLUSION}

We present a new approach for accounting incompressibility in the context of the natural element method (NEM), based on the definition of natural element bubbles approximation functions. In this variant of the NEM, which we refer as b-NEM, standard natural neighbour interpolation scheme is enriched with some bubble functions associated with the topological entities of the Delaunay tessellation of the set of nodes (edges or triangles in 2D), computed from the product of standard NEM shape functions related to the original nodes. In this manner the computation of these new shape functions does not imply additional costly computations. The mixed b-NEM/NEM approximation scheme has been tested in the context of 

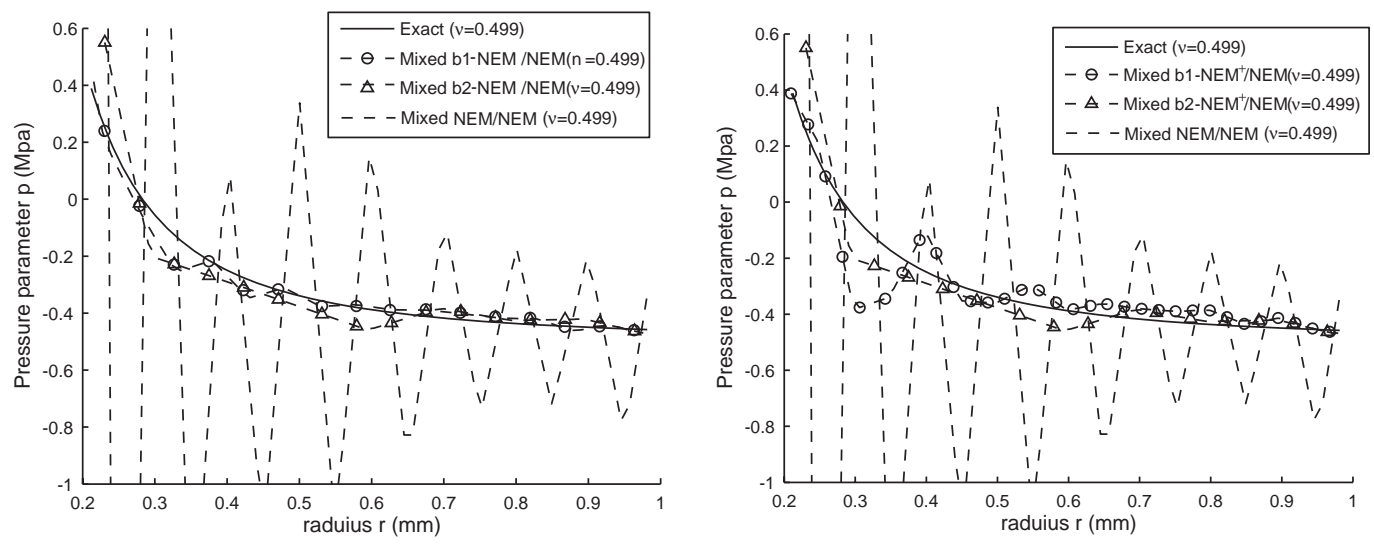

Figure 19. Exact and computed pressures $p$ along $\theta=0$ for $v=0.499$.

near-incompressible elastostatics. The numerical inf-sup test is satisfied and pressure oscillations are removed.

We have also corrected the resulting approximation schemes (b1-NEM or b2-NEM) enforcing some reproducing conditions (bilinear or quadratic) using the moving least square technique. In that case, the enriched approximations are noted by b1-NEM ${ }^{+}$and $\mathrm{b} 2-\mathrm{NEM}^{+}$. The technique just proposed allows higher approximation consistency than standard NEM, such as quadratic approximation for b2-NEM ${ }^{+}$. However, no significant improvement is noticed in the resolution convergence that remains first order, probably due to numerical integration, whose analysis is a work in progress.

\section{REFERENCES}

1. Bathe KJ. Finite Element Procedures. Prentice-Hall: Englewood Cliffs, NJ, 1986.

2. Girault V, Raviart P-A. Finite Element Methods for Navier-Stokes Equations. Theory and Algorithms. Springer: Berlin, 1986.

3. Donea J, Huerta A. Finite Element Methods for Flow Problems. Wiley: Chichester, 2003.

4. Brezzi F, Fortin M. Mixed and Hybrid Finite Element Methods. Springer: New York: 1991.

5. Malkus DS. Eigenproblems associated with the discrete LBB condition for the incompressible finite elements. International Journal of Engineering Science 1981; 10:1299-1310.

6. Chapelle D, Bathe K-J. The inf-sup test. Computers and Structures 1993; 48(5):745-760.

7. Nayroles B, Touzot G, Villon P. Generalizing the finite element method: diffuse approximation and diffuse elements. Computational Mechanics 1992; 10:307-318.

8. Belytschko T, Lu YY, Gu L. Element-free Galerkin methods. International Journal for Numerical Methods in Engineering 1994; 37:229-256.

9. Liu WK, Jun S, Zhang YF. Reproducing kernel particle methods. International Journal for Numerical Methods in Fluids 1995; 21:1081-1106.

10. Lucy LB. A numerical approach to the testing of fusion process. The Astronomic Journal 1977; 88: 1013-1024.

11. Sharan M, Kansa EJ, Gupta S. Application of the multiquadratic method for numerical solution of elliptic partial differential equations. Applied Mathematics and Computation 1997; 84:837-857.

12. Sukumar N, Moran B, Belytschko T. The natural elements method in solid mechanics. International Journal for Numerical Methods in Engineering 1998; 43:839-887.

13. Zhu T, Atluri SN. A modified collocation method and a penalty formulation for enforcing the essential boundary conditions in the element free Galerkin method. Computational Mechanics 1998; 21:211-222. 
14. Askes H, de Borst R, Heeres O. Conditions for locking-free elastoplastic analyses in the element-free Galerkin method. Computer Methods in Applied Mechanics and Engineering 1999; 173:99-109.

15. Chen JS, Pan C, Wu CT. Large deformation analysis of rubber based on a reproducing kernel particle method. Computer Methods in Applied Mechanics and Engineering 1997; 173:99-109.

16. Li S, Hoa W, Liu WK. Mesh-free simulations of shear banding in large deformation. International Journal of Solids and Structures 2000; 37:7183-7206.

17. Dolbow J, Belytschko T. Volumetric locking in the element-free Galerkin method. International Journal for Numerical Methods in Engineering 1999; 46:925-942.

18. Huerta A, Fernández-Méndez S. Locking in the incompressible limit for the element free Galerkin method. International Journal for Numerical Methods in Engineering 2001; 51(11):1361-1383.

19. Vidal Y, Villon P, Huerta A. Locking in the element free Galerkin. Communications in Numerical Methods in Engineering 2003; 19(9):725-735.

20. Chen JS, Yoon S, Whang HP, Liu WK. An improved reproducing kernel particle method for nearly incompressible finite elasticity solids. Computer Methods in Applied Mechanics and Engineering 2000; 181:117-145.

21. Yvonnet J, Ryckelynck D, Lorong P, Chinesta F. A new extension of the natural element method for non convex and discontinuous domains: the constrained natural element method (C-NEM). International Journal for Numerical Methods in Engineering 2004; 60:1451-1474.

22. Cueto E, Doblaré M, Gracia L. Imposing essential boundary conditions in the natural elements method by means of density-scaled alpha-shapes. International Journal for Numerical Methods in Engineering 2000; 49:519-546.

23. Yvonnet J, Chinesta F, Lorong P, Rynckelynck D. The constrained natural element method (C-NEM) for treating thermal models involving moving interfaces. International Journal of Thermal Sciences 2005; 44: $559-569$.

24. Yvonnet J, Lorong P, Ryckelynck D, Chinesta F. Simulating dynamic thermo-elastoplasticity in large transformations with adaptive refinement in the natural element method: application to shear banding. International Journal of Forming Processes 2005, in press.

25. Sukumar N. The natural element method in solid mechanics. Ph.D. Dissertation, Northwestern University, Evanston, IL, 1998.

26. González D, Cueto E, Doblaré M. Volumetric locking in natural neighbour Galerkin methods. International Journal for Numerical Methods in Engineering 2004; 61:611-632.

27. Melenk JM, Babuška I. The partition of unity finite element method: basic theory and applications. Computer Methods in Applied Mechanics and Engineering 1996; 4:289-314.

28. Yoo J, Moran B, Chen J-S. Stabilized conforming nodal integration in the natural-element method. International Journal for Numerical Methods in Engineering 1998; 60:861-890.

29. Sambridge M, Braun J, McQueen M. Geophysical parameterization and interpolation of irregular data using natural neighbours. Geophysical Journal International 1995; 122:837-857.

30. Sibson R. A vector identity for the Dirichlet tessellations. Mathematical Proceedings of the Cambridge Philosophical Society 1980; 87:151-155.

31. Hiyoshi H, Sugihara K. Improving continuity of Voronoi-based interpolation over Delaunay spheres. Computational Geometry 2002; 22:167-183.

32. Munkres JR. Elements of Algebraic Topology. Perseus Press, 1993; 7-14.

33. Zienkiewicz OC, De JP, Gago SR, Kelly DW. The hierarchical concept in finite element analysis. Computers and Structures 1983; 16:53-65.

34. Arnold DN, Brezzi F, Fortin M. A stable finite element for the Stockes equations. Calcolo 1984; 21: 337-344.

35. Piper B. Properties of local co-ordinates based on Dirichlet tessellations. Computing Supplement 1993; 8:227-239.

36. Hughes TJR. The Finite Element Method. Prentice-Hall: Englewood Cliffs, NJ, 1987.

37. Timoshenko SP, Goodier JN. Theory of Elasticity (3rd edn). McGraw-Hill: New York.

38. Szabó B, Bubuška I. Finite Element Analysis. Wiley: New York, 1991. 